\title{
Full field analysis of an anti-plane interface crack subjected to dynamic body forces
}

\author{
Chien-Ching $\mathrm{Ma}^{*}$, Kuo-Cheng Huang \\ Department of Mechanical Engineering, National Taiwan University, Taipei, Taiwan 10617, Republic of China
}

(Received 17 April 1997; in revised form 14 October 1997)

\begin{abstract}
In this study, the transient full field response of an interface crack between two different media subjected to dynamic body force at one material is investigated. For time $t<0$, the bimaterial medium is stress free and at rest. At $t=0$, a concentrated anti-plane dynamic point loading is applied at the medium as shown in Fig. 1. The total wave field is due to the effect of this point loading and the scattering of the incident waves by the interface crack. An alternative methodology that is different from the conventional superposition method is used to construct the reflected, refracted and diffracted wave fields. A useful fundamental solution is proposed in this study and the full field solution is determined by superposition of the fundamental solution in the Laplace transform domain. The proposed fundamental problem is the problem of applying an exponentially distributed traction (in the Laplace transform domain) on the interfacial crack faces. The Cagniard-de Hoop method of Laplace inversion is used to obtain the transient solution in time domain. Exact transient closed form solutions for stresses and stress intensity factors are obtained. Numerical results for the time history of stresses and stress intensity factors during the transient process are discussed in detail. (C) 1998 Elsevier Science Ltd. All rights reserved
\end{abstract}

\section{Introduction}

The interaction of a stress wave with a crack in an unbounded medium is a complicated problem. The analytical study of this problem is restricted to relatively simple cases for homogeneous material. Many structures are composed of different materials formed in layers for both man-made and natural origin. The layers are bonded together along interfaces. For the last two decades, the importance of composite materials has increased very rapidly in engineering applications because of their high strength and light weight. However, flaws contained at the interfaces of composite bodies due to improper adhesion may lead to serious danger, and a better understanding of interface fracture mechanics is needed. Since the inherent time dependence of a dynamic fracture

\footnotetext{
* Corresponding author.
} 
process results in mathematical models that are more complex than equivalent quasi-static models, most of the analyses done regarding cracked composite bodies are quasi-static. However, because of increasing applications of multilayered materials in modern engineering structures, there is still substantial interest in the dynamic interface fracture problem and many efforts should be added in this field in recent years.

The asymptotic elastic fields of a semi-infinite crack lying along an interface between dissimilar isotropic materials subjected to static loading was first considered by Williams (1959) for plane strain condition. A number of solutions for the stress and the displacement field near the crack tip are obtained by England (1965), Erdogan (1965) and Rice and Sih (1965). Extensions to anisotropic elasticity for the near tip field have been made by Gotoh (1967), Bogy (1972), and Kuo and Bogy (1974), and recently by Ting (1986, 1990), Qu and Bassani (1989). The exact full field solutions of interface cracks in anisotropic dissimilar media is obtained by Ma and Luo (1996).

The extension of an interface crack under the influence of transient horizontally polarized shear wave was analyzed by Brock and Achenbach (1973). It is assumed that the adhesive behaves as a perfectly plastic material, so that the stress in the zone of interface yielding is uniform and equal to the yield stress. Analytic solutions for the time of rupture and for the interface stress ahead of yield zone are obtained by applying integral transform methods. In conventional studies of a semiinfinite crack in an unbounded homogeneous medium subjected to a spatially uniform traction distribution acting on crack faces, the complete solution is obtained by integral transform methods together with a direct application of the Wiener-Hopf technique and the Cagniard-de Hoop method of Laplace inversion. The evolution of the Wiener-Hopf technique and a number of extensions are described by Noble (1958). The application of the technique to transient problems in elastodynamics was pioneered by de Hoop (1958) in a study of several diffraction problems. If the loading is replaced by a nonuniform distribution having a characteristic length, then the straightforward application of the Wiener-Hopf method is not successful. Freund (1974b) studied the problem of an elastic homogeneous solid containing a half-plane crack subjected to a pair of concentrated loadings on the crack faces at a finite distance from the crack tip. The exact solution of this problem was obtained by an indirect approach based on the superposition of moving dislocation. Basing his procedure on this method, Brock $(1982,1984)$, Brock et al. (1985) and Ma and Hou $(1990,1991)$ analyzed a series of problems of a semi-infinite crack in a homogeneous medium subjected to impact loading. In a series of papers, Freund (1972a, b, 1973, 1974a) developed important analytical methods for evaluating the transient field of a propagating crack. In Freund's papers, the difficult problem was separated into a number of more basic problems. By building up the so-called fundamental solutions of these basic-problems, the complete problem can then be obtained by superposition of the fundamental solution for the negation of the stress distribution on the prospective fracture plane. Based on the superposition method proposed by Freund, a series of problems for nonplanar crack propagation in a homogeneous medium was solved by Ma and Burgers (1986, 1987, 1988) and Ma $(1988,1990)$. For the aforementioned problems, either the direct application of the well-known Wiener-Hopf technique (Noble, 1958) is used or the superposition method proposed by Freund is performed to solve the problem. However, if a crack is subjected to incident non-planar waves, none of the known methods can be used directly to obtain the transient solutions.

In this study, the two-dimensional problem of a concentrated antiplane body force imposed suddenly near a semi-infinite crack is investigated in detail. At time $t=0$, an anti-plane con- 
centrated dynamic loadings is applied at the interface medium as shown in Fig. 1. This problem can be treated as the superposition of two problems. The first problem is the disturbance by a concentrated force in the crack-free, unbounded medium. The second problem considers the interface crack surfaces subjected to the negatives of the tractions induced by the first problem. To solve this problem, a new fundamental solution is proposed in this study and the complete solution is determined by superposition of the fundamental solution in the Laplace transform domain. The fundamental problem is the problem of applying an exponentially distributed traction on the interface crack faces in the Laplace transform domain. This alternative superposition scheme has also been used to solve many transient problems for the interaction of incident cylindrical waves with cracks in homogeneous medium successfully, e.g., Tsai and Ma (1992) for a stationary crack and Ma and Ing (1995) for a propagating crack. The transient full-field solutions of the interface crack and the dynamic stress intensity factor are obtained and are expressed in a closed form. Numerical results of stresses and dynamic stress intensity factors for the problem considered are evaluated and discussed in detail. The investigation of the idealized semi-infinite interface crack in this study can provide some information for an actual elastodynamic fracture problem. It should be noted that while the analysis has been carried out by assuming semi-infinite cracks, the results remain valid for a finite interface crack up until the time at which waves diffracted from the far tip reach the material point.

\section{The fundamental problem and fundamental solutions}

In this section, an alternative fundamental problem is proposed and the fundamental solution is obtained by using the Wiener-Hopf technique. The advantage is that the fundamental solution is easy to obtain and the complete solution is easy to construct by superposition of the fundamental solution. Consider a fundamental problem of anti-plane deformation for an interface crack in dissimilar materials subjected to dynamic loadings. Figure 1 shows the interface crack geometry and the coordinate systems. Materials 1 and 2 occupy the lower and upper half-spaces, respectively. The governing equations of wave motions in two media are

$$
\frac{\partial^{2} w^{(i)}}{\partial x^{2}}+\frac{\partial^{2} w^{(i)}}{\partial y^{2}}=b_{i}^{2} \frac{\partial^{2} w^{(i)}}{\partial t^{2}}, \quad i=1,2
$$

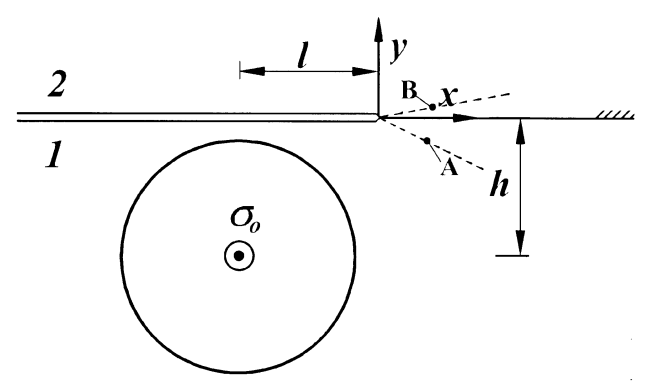

Fig. 1. The configuration and coordinate system of an interface crack in a bimaterial medium. 
where the superscript $i(i=1,2)$ refers to the lower and upper media, respectively; $w^{(i)}$ are the outof-plane displacements, and $b_{i}$ are the slownesses of the shear waves given by

$$
b_{i}=\frac{1}{c_{i}}=\sqrt{\frac{\rho_{i}}{\mu_{i}}}
$$

in which $c_{i}$ are the shear wave speeds, $\mu_{i}$ and $\rho_{i}$ are the respective shear moduli and the mass densities of two materials. The nonvanishing shear stresses are

$$
\sigma_{y z}^{(i)}=\mu_{i} \frac{\partial w^{(i)}}{\partial y}, \quad \sigma_{x z}^{(i)}=\mu_{i} \frac{\partial w^{(i)}}{\partial x} .
$$

The solution for an exponentially distributed loading applied at crack faces in the Laplace transform domain will be referred to as the fundamental solution. Then boundary conditions on crack surfaces expressed in the Laplace transform domain can be described as follows

$$
\bar{\sigma}_{y z}^{(1)}(x, 0, p)=\bar{\sigma}_{y z}^{(2)}(x, 0, p)=e^{p \eta x}, \quad-\infty<x<0,
$$

where $p$ is the Laplace transform parameter and $\eta$ is a constant. The overbar symbol is used for denoting the transform on time $t$. The one-sided Laplace transform with respect to time and the two-sided Laplace transform with respect to $x$ are defined by

$$
\begin{aligned}
& \bar{w}(x, y, p)=\int_{0}^{\infty} w(x, y, t) e^{-p t} \mathrm{~d} t, \\
& \tilde{w}(\lambda, y, p)=\int_{0}^{\infty} \bar{w}(x, y, p) e^{-p \lambda x} \mathrm{~d} x .
\end{aligned}
$$

The displacements and shear stresses must be continuous on the interface which gives the following conditions on the interface

$$
\begin{array}{ll}
\bar{\sigma}_{y z}^{(1)}(x, 0, p)=\bar{\sigma}_{y z}^{(2)}(x, 0, p) & 0<x<\infty, \\
\bar{w}^{(1)}(x, 0, p)=\bar{w}^{(2)}(x, 0, p) & 0<x<\infty .
\end{array}
$$

The solution of the proposed fundamental problem can be obtained by using integral transform methods. Apply a one-sided Laplace transform with respect to $t$ and a two-sided Laplace transform with respect to $x$ on (1). General solutions in the transform domain, which are bounded as $y \rightarrow$ $-\infty$ (and $+\infty$, respectively), can be expressed as

$$
\begin{aligned}
& \tilde{w}^{(1)}(\lambda, y, p)=A_{1}(p, \lambda) e^{p \alpha_{1}(\lambda) y}, \\
& \tilde{w}^{(2)}(\lambda, y, p)=A_{2}(p, \lambda) e^{-p \alpha_{2}(\lambda) y},
\end{aligned}
$$

where

$$
\alpha_{i}(\lambda)=\sqrt{b_{i}+\lambda} \sqrt{b_{i}-\lambda}=\alpha_{i}^{+}(\lambda) \alpha_{i}^{-}(\lambda), \quad i=1,2
$$

and $A_{1}, A_{2}$ are unknown functions. The branch cuts of $\alpha_{i}$ are introduced to ensure $\operatorname{Re}\left(\alpha_{i}\right) \geqslant 0$ in the entire cut complex $\lambda$-plane, where "Re" denotes the real part. 
Application of the Laplace transforms to the boundary conditions (3)-(5) yields

$$
\begin{aligned}
& \bar{\sigma}_{y z}^{(1)}(\lambda, 0, p)=\bar{\sigma}_{y z}^{(2)}(\lambda, 0, p)=\frac{1}{p(\eta-\lambda)}+\bar{\sigma}_{y z}^{+}, \quad-\infty<x<\infty \\
& \tilde{w}^{(1)}(\lambda, 0, p)=\tilde{w}^{(2)}(\lambda, 0, p)=A^{+}, \quad 0<x<\infty
\end{aligned}
$$

where $\tilde{\sigma}_{y z}^{+}$and $A^{+}$are unknown functions.

From eqns (6) and (7), the transformed displacements and shear stresses along the interface crack line $y=0$ are

$$
\begin{aligned}
& \tilde{w}^{(1)}(\lambda, 0, p)=A_{1}=A^{+}+A_{1}^{-}, \\
& \tilde{w}^{(2)}(\lambda, 0, p)=A_{2}=A^{+}+A_{2}^{-}, \\
& \mu_{1} p \alpha_{1}(\lambda) A_{1}=-\mu_{2} p \alpha_{2}(\lambda) A_{2}=\frac{1}{p(\eta-\lambda)}+\tilde{\sigma}_{y z}^{+} .
\end{aligned}
$$

In eqns (11) and (12), $A_{1}^{-}$and $A_{2}^{-}$are unknown functions analytic in $\operatorname{Re}(\lambda)<b_{i}$, respectively. Eliminating $A^{+}$through (11)-(13), we have

$$
A^{-}=\frac{\mu_{1} \alpha_{1}(\lambda)+\mu_{2} \alpha_{2}(\lambda)}{p \mu_{1} \mu_{2} \alpha_{1}(\lambda) \alpha_{2}(\lambda)}\left[\frac{1}{p(\eta-\lambda)}+\tilde{\sigma}_{y z}^{+}\right]
$$

where $A^{-}=A_{1}^{-}-A_{2}^{-}$is the transformed crack-opening displacement.

Case 1: $b_{1}>b_{2}$

At this point it is convenient to introduce a new function $S_{2}(\lambda)$ by defining

$$
S_{2}(\lambda)=\frac{\mu_{1} \alpha_{1}(\lambda)+\mu_{2} \alpha_{2}(\lambda)}{\left(\mu_{1}+\mu_{2}\right) \alpha_{1}(\lambda)} .
$$

The function $S_{2}(\lambda)$ has the properties that $S_{2}(\lambda) \rightarrow 1$ as $|\lambda| \rightarrow \infty$, and that $S_{2}(\lambda)$ has neither zeros nor poles in the $\lambda$-plane by cuts along $b_{2}<\lambda<b_{1}$ and $-b_{1}<\lambda<-b_{2}$. From the general product factorization method, $S_{2}(\lambda)$ can be written as the product of two regular functions $S_{2}^{+}(\lambda)$ and $S_{2}^{-}(\lambda)$, where

$$
S_{2}^{ \pm}(\lambda)=\exp \left\{-\frac{1}{\pi} \int_{b_{2}}^{b_{1}} \tan ^{-1}\left[\frac{\mu_{2}\left(\delta_{2}^{2}-b_{2}^{2}\right)^{1 / 2}}{\mu_{1}\left(b_{1}^{2}-\delta^{2}\right)^{1 / 2}}\right] \frac{1}{\delta \pm \lambda} \mathrm{d} \delta\right\} .
$$

In view of the previous discussion, eqn (14) may be rewritten as

$$
\begin{aligned}
& p \sqrt{b_{2}-\lambda} \frac{A^{-}}{S_{2}^{-}(\lambda)}-\frac{\mu_{1}+\mu_{2}}{p \mu_{1} \mu_{2}(\eta-\lambda)} \frac{S_{2}^{+}(\eta)}{\sqrt{b_{2}+\eta}} \\
&=\frac{\left(\mu_{1}+\mu_{2}\right)}{\sqrt{b_{2}+\lambda}} \frac{S_{2}^{+}(\lambda)}{\mu_{1} \mu_{2}} \tilde{\sigma}_{y z}^{+}+\frac{\mu_{1}+\mu_{2}}{p \mu_{1} \mu_{2}(\eta-\lambda)}\left[\frac{S_{2}^{+}(\lambda)}{\sqrt{b_{2}+\lambda}}-\frac{S_{2}^{+}(\eta)}{\sqrt{b_{2}+\eta}}\right] .
\end{aligned}
$$

The left-hand side of this equation is regular for $\operatorname{Re}(\lambda)<b_{2}$, while the right-hand side is regular 
for $\operatorname{Re}(\lambda)>-b_{2}$. Applying the analytic continuation argument, therefore, each side of eqn (17) represents one and the same entire function, say $E(\lambda)$. By Liouville's theorem, the bounded entire function $E(\lambda)$ is a constant. The magnitude of the constant can be obtained from order conditions on $E(\lambda)$ as $|\lambda| \rightarrow \infty$, which in turn are obtained from order conditions on the dependent field variables in the vicinity of $x=0$. Furthermore, $\bar{w}_{-}^{(1)}(x, 0, p)-\bar{w}_{-}^{(2)}(x, 0, p)$ is expected to vanish as $x \rightarrow 0^{-}$to ensure continuity of displacement, and $\bar{\sigma}_{y z}^{+}(x, 0, p)$ is expected to be square root singular as $x \rightarrow 0^{+}$. Consequently from the Abel theorem, $E(\lambda)$ vanishes completely and then from eqn (17), we find

$$
A^{-} \equiv A_{1}^{-}-A_{2}^{-}=\frac{\left(\mu_{1}+\mu_{2}\right) S_{2}^{+}(\eta) S_{2}^{-}(\lambda)}{p^{2} \mu_{1} \mu_{2} \sqrt{b_{2}-\lambda} \sqrt{b_{2}+\eta}} \frac{1}{\eta-\lambda} .
$$

Making use of eqns (11)-(13) and eliminating $\tilde{\sigma}_{y z}^{+}$, we obtain

$$
A^{+}=-\left(\frac{\mu_{1} \alpha_{1} A_{1}^{-}}{\mu_{1} \alpha_{1}+\mu_{2} \alpha_{2}}+\frac{\mu_{2} \alpha_{2} A_{2}^{-}}{\mu_{1} \alpha_{1}+\mu_{2} \alpha_{2}}\right) \text {. }
$$

Substituting $A_{2}^{-}$from (18) into (19), $A_{1}$ can be found as

$$
A_{1}=\frac{S_{2}^{+}(\eta) \sqrt{b_{2}+\lambda}}{p^{2} S_{2}^{+}(\lambda) \mu_{1} \alpha_{1} \sqrt{b_{2}+\eta}(\eta-\lambda)} .
$$

Similarly substituting $A_{1}^{-}$from (18) into (19), we have

$$
A_{2}=\frac{-S_{2}^{+}(\eta)}{p^{2} S_{2}^{+}(\lambda) \mu_{2} \sqrt{b_{2}+\eta} \sqrt{b_{2}-\lambda}(\eta-\lambda)} .
$$

In view of eqns (20), (21), (6) and (7), inverting the two-sided Laplace transform, we obtain the fundamental solutions of stresses and displacements for the fundamental problem in the Laplace transform domain as follows

$$
\begin{aligned}
& \bar{\sigma}_{y z}^{(1)}(x, y, p)=\frac{1}{2 \pi i} \int \frac{S_{2}^{+}(\eta) \sqrt{b_{2}+\lambda}}{S_{2}^{+}(\lambda) \sqrt{b_{2}+\eta}(\eta-\lambda)} e^{p\left[\alpha_{1}(\lambda) y+\lambda x\right]} \mathrm{d} \lambda, \\
& \bar{\sigma}_{x z}^{(1)}(x, y, p)=\frac{1}{2 \pi i} \int \frac{S_{2}^{+}(\eta) \sqrt{b_{2}+\lambda} \lambda}{S_{2}^{+}(\lambda) \alpha_{1}(\lambda) \sqrt{b_{2}+\eta}(\eta-\lambda)} e^{p\left[\alpha_{1}(\lambda) y+\lambda x\right]} \mathrm{d} \lambda, \\
& \bar{w}^{(1)}(x, y, p)=\frac{1}{2 \pi i} \int \frac{S_{2}^{+}(\eta) \sqrt{b_{2}+\lambda}}{\mu_{1} p S_{2}^{+}(\lambda) \alpha_{1}(\lambda) \sqrt{b_{2}+\eta}(\eta-\lambda)} e^{p\left[\alpha_{1}(\lambda) y+\lambda x\right]} \mathrm{d} \lambda, \\
& \bar{\sigma}_{y z}^{(2)}(x, y, p)=\frac{1}{2 \pi i} \int \frac{S_{2}^{+}(\eta) \sqrt{b_{2}+\lambda}}{S_{2}^{+}(\lambda) \sqrt{b_{2}+\eta}(\eta-\lambda)} e^{-p\left[\alpha_{2}(\lambda) y-\lambda x\right]} \mathrm{d} \lambda, \\
& \bar{\sigma}_{x z}^{(2)}(x, y, p)=\frac{1}{2 \pi i} \int \frac{-S_{2}^{+}(\eta) \lambda}{S_{2}^{+}(\lambda) \sqrt{b_{2}+\eta} \sqrt{b_{2}-\lambda}(\eta-\lambda)} e^{-p\left[\alpha_{2}(\lambda) y-\lambda x\right]} \mathrm{d} \lambda,
\end{aligned}
$$




$$
\bar{w}^{(2)}(x, y, p)=\frac{1}{2 \pi i} \int \frac{-S_{2}^{+}(\eta)}{\mu_{2} p S_{2}^{+}(\lambda) \sqrt{b_{2}+\eta} \sqrt{b_{2}-\lambda}(\eta-\lambda)} e^{-p\left[\alpha_{2}(\lambda) y-\lambda x\right]} \mathrm{d} \lambda .
$$

The corresponding result of the dynamic stress intensity factor expressed in the Laplace transform domain is

$$
\begin{aligned}
\bar{K}_{\mathrm{III}}(p, \eta) & =\lim _{x \rightarrow 0} \sqrt{2 \pi x} \bar{\sigma}_{y z}(x, 0, p) \\
& =\frac{-(2 / \pi)^{1 / 2} S_{2}^{+}(\eta)}{\left(b_{2}+\eta\right)^{1 / 2}} \int_{0}^{\infty} \frac{e^{-p t}}{t^{1 / 2}} \mathrm{~d} t=\frac{\sqrt{2} S_{2}^{+}(\eta)}{\left(b_{2}+\eta\right)^{1 / 2} p^{1 / 2}} .
\end{aligned}
$$

Case 2: $b_{1}<b_{2}$

Following the similar procedure as presented previously for case $1\left(b_{1}>b_{2}\right)$, we have

$$
\begin{aligned}
& A_{1}=\frac{S_{1}^{+}(\eta)}{p^{2} S_{1}^{+}(\lambda) \mu_{1} \sqrt{b_{1}+\eta} \sqrt{b_{1}-\lambda}(\eta-\lambda)}, \\
& A_{2}=\frac{-S_{1}^{+}(\eta) \sqrt{b_{1}+\lambda}}{p^{2} S_{1}^{+}(\lambda) \mu_{2} \alpha_{2} \sqrt{b_{1}+\eta}(\eta-\lambda)},
\end{aligned}
$$

where

$$
S_{1}^{ \pm}(\lambda)=\exp \left\{-\frac{1}{\pi} \int_{b_{1}}^{b_{2}} \tan ^{-1}\left[\frac{\mu_{1}\left(\delta^{2}-b_{1}^{2}\right)^{1 / 2}}{\mu_{2}\left(b_{2}^{2}-\delta^{2}\right)^{1 / 2}}\right] \frac{1}{\delta \pm \lambda} \mathrm{d} \delta\right\} .
$$

The solutions of stresses and displacements for the fundamental problem in the Laplace transform domain are

$$
\begin{aligned}
& \bar{\sigma}_{y z}^{(1)}(x, y, p)=\frac{1}{2 \pi i} \int \frac{S_{1}^{+}(\eta) \sqrt{b_{1}+\lambda}}{S_{1}^{+}(\lambda) \sqrt{b_{1}+\eta}(\eta-\lambda)} e^{p\left[\alpha_{1}(\lambda) y+\lambda x\right]} \mathrm{d} \lambda, \\
& \bar{\sigma}_{x z}^{(1)}(x, y, p)=\frac{1}{2 \pi i} \int \frac{\lambda S_{1}^{+}(\eta)}{S_{1}^{+}(\lambda) \sqrt{b_{1}+\eta} \sqrt{b_{1}-\lambda}(\eta-\lambda)} e^{p\left[\alpha_{1}(\lambda) y+\lambda x\right]} \mathrm{d} \lambda, \\
& \bar{w}^{(1)}(x, y, p)=\frac{1}{2 \pi i} \int \frac{S_{1}^{+}(\eta)}{\mu_{1} p S_{1}^{+}(\lambda) \sqrt{b_{1}+\eta} \sqrt{b_{1}-\lambda}(\eta-\lambda)} e^{p\left[\alpha_{1}(\lambda) y+\lambda x\right]} \mathrm{d} \lambda, \\
& \bar{\sigma}_{y z}^{(2)}(x, y, p)=\frac{1}{2 \pi i} \int \frac{S_{1}^{+}(\eta) \sqrt{b_{1}+\lambda}}{S_{1}^{+}(\lambda) \sqrt{b_{1}+\eta(\eta-\lambda)}} e^{-p\left[\alpha_{2}(\lambda) y-\lambda x\right]} \mathrm{d} \lambda, \\
& \bar{\sigma}_{x z}^{(2)}(x, y, p)=\frac{1}{2 \pi i} \int \frac{-\lambda S_{1}^{+}(\eta) \sqrt{b_{1}+\lambda}}{S_{1}^{+}(\lambda) \alpha_{2}(\lambda) \sqrt{b_{1}+\eta}(\eta-\lambda)} e^{-p\left[\alpha_{2}(\lambda) y-\lambda x\right]} \mathrm{d} \lambda,
\end{aligned}
$$




$$
\bar{w}^{(2)}(x, y, p)=\frac{1}{2 \pi i} \int \frac{-S_{1}^{+}(\eta) \sqrt{b_{1}+\lambda}}{\mu_{2} p S_{1}^{+}(\lambda) \alpha_{2}(\lambda) \sqrt{b_{1}+\eta}(\eta-\lambda)} e^{-p\left[\alpha_{2}(\lambda) y-\lambda x\right]} \mathrm{d} \lambda .
$$

The corresponding result of the stress intensity factor is

$$
\bar{K}_{\mathrm{III}}(p, \eta)=\frac{-\sqrt{2} S_{1}^{+}(\eta)}{\left(b_{1}+\eta\right)^{1 / 2} p^{1 / 2}}
$$

\section{Transient full field solution in time domain}

As shown in Fig. 1, a bimaterial medium is composed of two homogeneous, isotropic, and linearly elastic solids. Materials 1 and 2 occupy the lower and upper half-planes, respectively. A semi-infinite crack lying along the interface of the bimaterial is initially stress-free and at rest. At time $t=0$, a concentrated anti-plane dynamic loading with magnitude $\sigma_{0}$ is applied at the lower half-plane $x=-l$ and $y=-h$ in material 1 . The two-dimensional geometry of the problem is shown in Fig. 1. The time dependence of the concentrated loading is represented by the Heaviside step function $H(t)$. Dynamic stress intensity factor will be induced as the incident cylindrical wave generated from the point loading arrives at the crack tip. After the incident cylindrical wave interaction with the semi-infinite crack and the interface, diffracted and reflected waves will be generated from the crack tip, and refracted waves will be induced from the interface.

The incident field of the cylindrical wave generated by the concentrated loading expressed in the Laplace transform domain can be represented as follows

$$
\bar{\sigma}_{y z}^{i}(x, y, s)=\frac{1}{2 \pi i} \int-\sigma_{0} e^{-p\left[\alpha_{1}(y+h)-\eta(x+l)\right]} \mathrm{d} \eta .
$$

The applied traction on the crack faces as indicated in (39), has the functional form $e^{p \eta x}$. Since the solutions of applying traction $e^{p \eta x}$ on crack faces have been solved in the previous section, the scattering field generated from the semi-infinite crack can be constructed by superimposing the incident wave traction that is equal to (39). When we combine (22), (25) and (39), the stress fields $\sigma_{y z}$ for lower and upper planes expressed in the Laplace transform domain can be obtained as follows

Case 1: $b_{1}>b_{2}$

$$
\begin{aligned}
\bar{\sigma}_{y z}^{(1)}=\frac{\sigma_{0}}{2 \pi i} \int_{\Gamma_{\eta}}\left\{-e^{-p\left[\alpha_{1}(y+h)-\eta(x+l)\right]}+r_{1 / 2}(\eta) e^{p\left[\alpha_{1}(y-h)+\eta(x+l)\right]}\right. \\
\left.+\frac{f_{1 / 2}(\eta)}{2 \pi i} \int_{\Gamma_{\lambda}} \frac{S_{2}^{+}(\eta) \sqrt{b_{2}+\lambda} e^{p\left[\alpha_{1}(\lambda) y+\lambda x\right]}}{S_{2}^{+}(\lambda) \sqrt{b_{2}+\eta}(\eta-\lambda)} \mathrm{d} \lambda \cdot e^{-p \alpha_{1}(\eta) h+p \eta l}\right\} \mathrm{d} \eta,
\end{aligned}
$$




$$
\begin{aligned}
\bar{\sigma}_{y z}^{(2)}=\frac{\sigma_{0}}{2 \pi i} \int_{\Gamma_{\eta}}\left\{-f_{1 / 2}(\eta) e^{p\left[\alpha_{2} y-\alpha_{1} h+\eta(x+l)\right]}\right. & \\
& \left.+\frac{f_{1 / 2}(\eta)}{2 \pi i} \int_{\Gamma_{\lambda}} \frac{S_{2}^{+}(\eta) \sqrt{b_{2}+\lambda} e^{-p\left[\alpha_{2}(\lambda) y-\lambda x\right]}}{S_{2}^{+}(\lambda) \sqrt{b_{2}+\eta}(\eta-\lambda)} \mathrm{d} \lambda \cdot e^{-p \alpha_{1}(\eta) h+p \eta l}\right\} \mathrm{d} \eta .
\end{aligned}
$$

Case 2: $b_{1}<b_{2}$

$$
\begin{array}{r}
\bar{\sigma}_{y z}^{(1)}=\frac{\sigma_{0}}{2 \pi i} \int_{\Gamma_{\eta}}\left\{-e^{-p\left[\alpha_{1}(y+h)-\eta(x+l)\right]}+r_{1 / 2}(\eta) e^{p\left[\alpha_{1}(y-h)+\eta(x+l)\right]}\right. \\
\left.+\frac{f_{1 / 2}(\eta)}{2 \pi i} \int_{\Gamma_{\lambda}} \frac{S_{1}^{+}(\eta) \sqrt{b_{1}+\lambda} e^{p\left[\alpha_{1}(\lambda) y+\lambda x\right]}}{S_{1}^{+}(\lambda) \sqrt{b_{1}+\eta}(\eta-\lambda)} \mathrm{d} \lambda \cdot e^{-p \alpha_{1}(\eta) h+p \eta l}\right\} \mathrm{d} \eta, \\
\bar{\sigma}_{y z}^{(2)}=\frac{\sigma_{0}}{2 \pi i} \int_{\Gamma_{\eta}}\left\{-f_{1 / 2}(\eta) e^{p\left[\alpha_{2} y-\alpha_{1} h+\eta(x+l)\right]}\right. \\
\left.+\frac{f_{1 / 2}(\eta)}{2 \pi i} \int_{\Gamma_{\lambda}} \frac{S_{1}^{+}(\eta) \sqrt{b_{1}+\lambda} e^{-p\left[\alpha_{2}(\lambda) y-\lambda x\right]}}{S_{1}^{+}(\lambda) \sqrt{b_{1}+\eta}(\eta-\lambda)} \mathrm{d} \lambda \cdot e^{-p \alpha_{1}(\eta) h+p \eta l}\right\} \mathrm{d} \eta,
\end{array}
$$

where

$$
\begin{aligned}
& r_{1 / 2}(\eta)=\frac{\mu_{1} \alpha_{1}(\eta)-\mu_{2} \alpha_{2}(\eta)}{\mu_{1} \alpha_{1}(\eta)+\mu_{2} \alpha_{2}(\eta)}, \\
& f_{1 / 2}(\eta)=\frac{2 \mu_{2} \alpha_{2}(\eta)}{\mu_{1} \alpha_{1}(\eta)+\mu_{2} \alpha_{2}(\eta)} .
\end{aligned}
$$

The first two terms in (40) [and (42)] represent the solution of incident and reflected waves in material 1 for applying a dynamic point loading in a bimaterial medium without crack, and the first term in (41) [and (43)] represents the refracted wave in material 2. The last term in (40)-(43) represents the waves generated due to the disturbance of the interface crack.

Equations (40)-(43) constitute a double inversion integral where the paths $\Gamma_{\lambda}$ and $\Gamma_{\eta}$ refer to Laplace inversion contours in the $\lambda$-plane and the $\eta$-plane, respectively. Here we shall carry out the inverse transform using an extension of the Cagniard-de Hoop technique. This technique enables two successive inversions to be performed in one step. In this particular problem, we use the technique proposed by Harris (1980) to perform three successive inversions. This technique was also used successfully by Tsai and Ma (1992) to solve the in-plane problem of a semi-infinite crack in a homogeneous medium subjected to dynamic concentrated forces. We introduce Cagniard contours in both the $\eta$ and $\lambda$-plane for material 1 by setting

$$
\begin{aligned}
& \alpha_{1}(\eta) h-\eta l=t_{p}, \\
& -\alpha_{1}(\lambda) y-\lambda x=t_{d_{1}} .
\end{aligned}
$$

Equations (44) and (45) can be solved for $\eta$ and $\lambda$ to yield 


$$
\begin{aligned}
& \eta^{ \pm}=\frac{-t_{p} \cos \theta_{p}}{R_{p}} \pm i \frac{\sin \theta_{p}}{R_{p}}\left(t_{p}^{2}-b_{1}^{2} R_{p}^{2}\right)^{1 / 2}, \\
& \lambda^{ \pm}=\frac{-t_{d_{p}} \cos \theta_{d_{1}}}{R_{d_{1}}} \pm i \frac{\sin \theta_{d_{1}}}{R_{d_{1}}}\left(t_{d_{1}}^{2}-b_{1}^{2} R_{d_{1}}^{2}\right)^{1 / 2},
\end{aligned}
$$

where

$$
R_{p}=\left(l^{2}+h^{2}\right)^{1 / 2}, \quad \theta_{p}=\cos ^{-1}\left(\frac{l}{R_{p}}\right) ; \quad R_{d_{1}}=\left(x^{2}+y^{2}\right)^{1 / 2}, \quad \theta_{d_{1}}=\cos ^{-1}\left(\frac{x}{R_{d_{1}}}\right)
$$

in which $\left(R_{p}, \theta_{p}\right)$ and $\left(R_{d_{1}}, \theta_{d_{1}}\right)$ are the polar coordinates of the source point and the field point in material 1, respectively. For material 2 , we set the Cagniard contour in the $\lambda$-plane

$$
\alpha_{2}(\lambda) y-\lambda x=t_{d_{2}} \text {. }
$$

Solve for $\lambda$ to yield

$$
\lambda^{ \pm}=\frac{-t_{d_{2}} \cos \theta_{d_{2}}}{R_{d_{2}}} \pm i \frac{\sin \theta_{d_{2}}}{R_{d_{2}}}\left(t_{d_{2}}^{2}-b_{2}^{2} R_{d_{2}}^{2}\right)^{1 / 2},
$$

where

$$
R_{d_{2}}=\left(x^{2}+y^{2}\right)^{1 / 2}, \quad \theta_{d_{2}}=\cos ^{-1}\left(\frac{x}{R_{d_{2}}}\right) .
$$

In the $\eta$-plane (or $\lambda$-plane), (46) [or (47), (49)] describes a hyperbola which is denoted as the Cagniard contour. We shift the $\eta$ and $\lambda$-integrations onto Cagniard contours along which $t_{p}, t_{d_{1}}$ and $t_{d_{2}}$ are both real and positive. In this technique, the two Cagniard contours must be superimposed in order to determine how and when they overlap for different locations of source and field points. Depending on this, the reflected, refracted and diffracted waves can be constructed automatically.

The last term in (40)-(43) has a functional form possesses a pole at $\eta=\lambda$, the contribution of the pole has to be taken into account in the change of integral paths from $\eta$ to $t_{p}$ and $\lambda$ to $t_{d_{1}}$. Consider the deformed integral contour shown in Fig. 2a, the requirement of $\operatorname{Re}(\eta)>\operatorname{Re}(\lambda)$ dictates that the integral path $\Gamma_{\eta}$ is always located at the right-hand side of $\Gamma_{\lambda}$. Recall that a pole term arises, representing the reflected waves generated from the crack face. For case $1\left(b_{1}>b_{2}\right)$ and at material 1, two material points are chosen to explain the generation of a reflected wave which represent the contour integral path (1) and (2) in Fig. 2a. After the change of the $\Gamma_{\eta}$ and $\Gamma_{\lambda}$ integrations onto Cagniard contours, a pole at $\eta=\lambda$ will be embraced for path (2) but no pole should be taken into account for path (1). Some important situations and generation of wave fronts are expressed in Tables 1 and 2 for case $1\left(b_{1}>b_{2}\right)$ and case $2\left(b_{1}<b_{2}\right)$, respectively. The corresponding contour integrals for case 1 and case 2 are represented in Fig. 2a-f and Fig. 3a-d, respectively. Complete wave fronts for some interesting cases are shown in Figs 4-9. The waves shown in Figs 4-9 are composed of incident wave, reflected wave, refracted wave, diffracted wave and head wave and are denoted by $I, R, F, D$ and $H$ (or $h$ ), respectively.

Some interesting phenomena of the head wave and refracted wave can be explained by the 

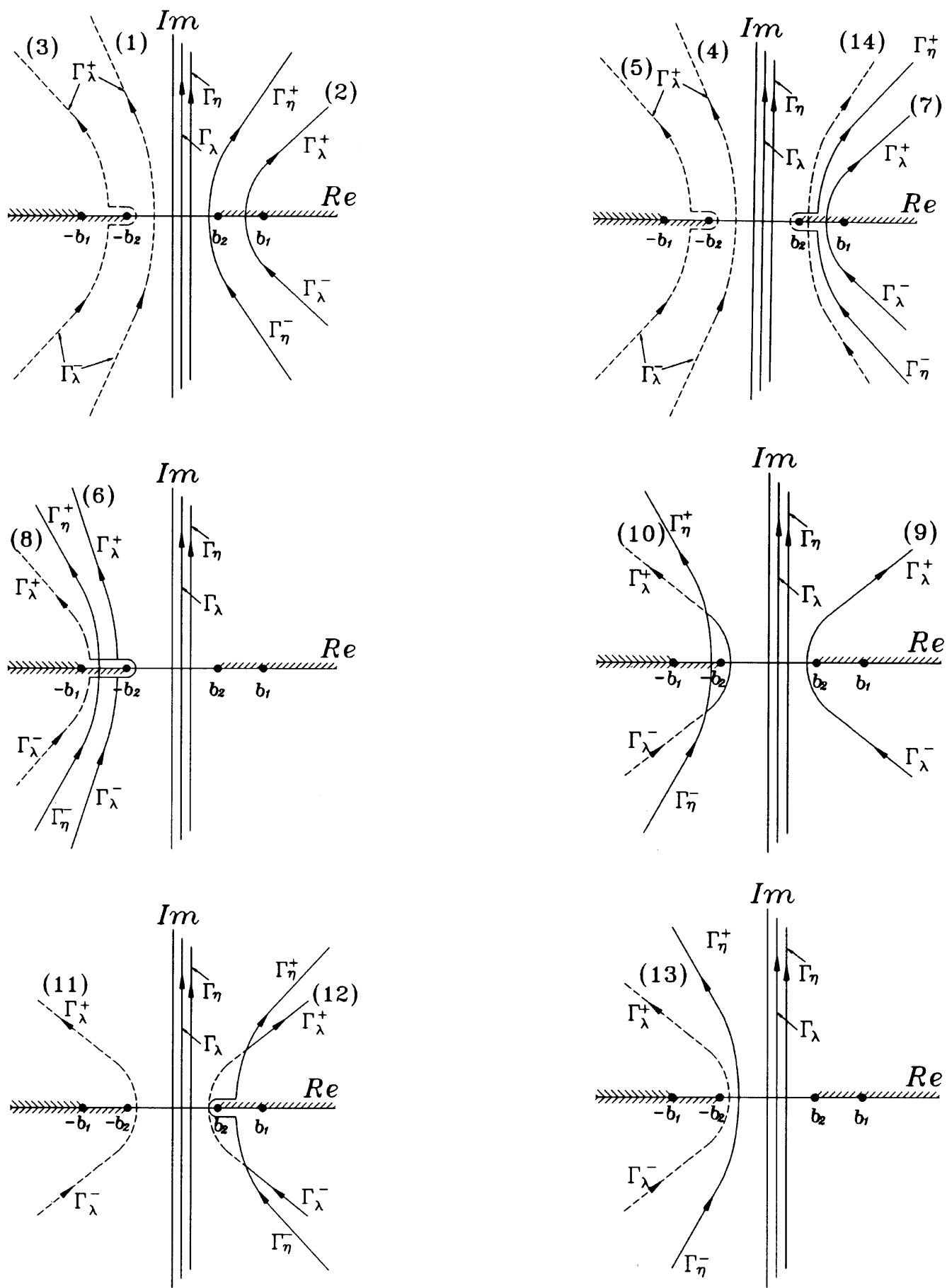

Fig. 2. (a) Deformed integral contour for conditions (1), (2) and (3) of case 1. (b) Deformed integral contour for conditions (4), (5), (7) and (14) of case 1. (c) Deformed integral contour for conditions (6) and (8) of case 1. (d) Deformed integral contour for conditions (9) and (10) of case 1. (e) Deformed integral contour for conditions (11) and (12) of case 1. (f) Deformed integral contour for condition (13) and of case 1. 
Table 1

Wave fronts generated in material 1 and material 2 for different conditions of case 1

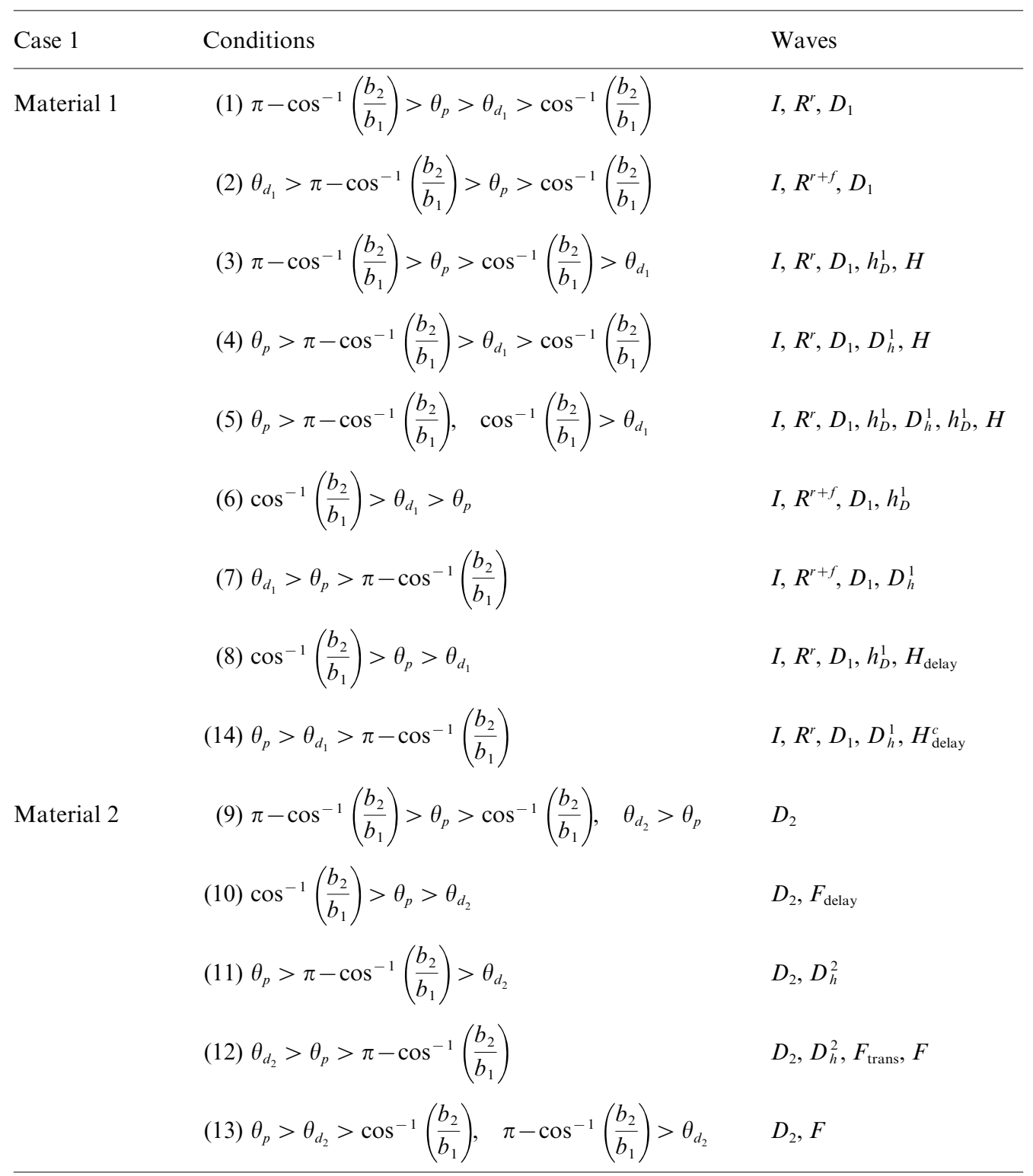

deformed integral contours as shown in Fig. 2b, $d$ and e. Consider the deformed contour path (14) as shown in Fig. 2b, the most important phenomenon is the distortion of the straight head wave

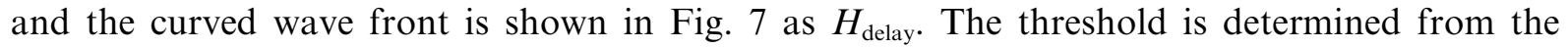
intersection of $\Gamma_{\eta}^{+}$and $\Gamma_{\lambda}^{+}$, indicating that the straight wave front of the head wave will be deformed and terminated at the wave front of $D_{1}$ wave. Next consider the deformed contour path (10) as 
Table 2

Wave fronts generated in material 1 and material 2 for different conditions of case 2

\begin{tabular}{lll}
\hline Case 2 & Conditions & Waves \\
\hline Material 1 & (1) $\theta_{p}>\theta_{d_{1}}$ & $I, R^{r}, D_{1}$ \\
(2) $\theta_{d_{1}}>\theta_{p}$ & $I, R^{r+f}, D_{1}$ \\
Material 2 & (3) $\theta_{p}>\theta_{d_{2}}>\cos ^{-1}\left(\frac{b_{1}}{b_{2}}\right), \frac{\pi}{2}>\theta_{p}$ & $D_{2}, F$ \\
& (4) $\theta_{d_{2}}>\frac{\pi}{2}>\theta_{p}$ & $D_{2}$ \\
(5) $\theta_{p}>\theta_{d_{2}}, \cos ^{-1}\left(\frac{b_{1}}{b_{2}}\right)>\theta_{d_{2}}$ & $D_{2}, h_{D}^{2}, F$ \\
(6) $\cos ^{-1}\left(\frac{b_{1}}{b_{2}}\right)>\theta_{d_{2}}>\theta_{p}$ & $D_{2}, F, F_{\text {trans }}$ \\
\hline
\end{tabular}

shown in Fig. 2d, two Cagniard contours overlap and this overlap would cause the refracted $F$ wave to distort which becomes $F_{\text {delay }}$ wave as shown in Fig. 4 . Finally, consider the deformed contour path (12) as shown in Fig. 2e, the overlap of these two Cagniard contours occurs and the integration path represented by the dashed line will terminate at the intersection point of these two contours. This indicates that the refracted $F$ wave is not complete and only exists before the extended $F_{\text {tran }}$ wave from the crack tip arrive.

The solutions of diffracted and head waves can be constructed by using the Cagniard-de Hoop technique. Finally the complete solutions of incident wave, reflected wave, refracted wave and diffracted wave expressed in time domain for shear stress $\sigma_{y z}$ are shown in the Appendix.

The corresponding dynamic stress intensity factors in time domain can be obtained as follows:

Case 1: $b_{1}>b_{2}$

(1) $\theta_{p}>\pi-\cos ^{-1}\left(b_{2} / b_{1}\right)$

$$
K_{\mathrm{III}}(t)=\sqrt{\frac{2}{\pi^{3}}} \int_{b_{1} R_{p}}^{t} \operatorname{Im}\left[\frac{f_{1 / 2}(\eta) S_{2}^{+}(\eta)}{\left(b_{2}+\eta\right)^{1 / 2}} \frac{\partial \eta}{\partial \tau}\right] \frac{1}{(t-\tau)^{1 / 2}} \mathrm{~d} \tau,
$$

(2) $\theta_{p}<\pi-\cos ^{-1}\left(b_{2} / b_{1}\right)$

$$
K_{\mathrm{III}}(t)=\sqrt{\frac{2}{\pi^{3}}} \int_{T_{h_{p}}}^{t} \operatorname{Im}\left[\frac{f_{1 / 2}(\eta) S_{2}^{+}(\eta)}{\left(b_{2}+\eta\right)^{1 / 2}} \frac{\partial \eta}{\partial \tau}\right] \frac{1}{(t-\tau)^{1 / 2}} \mathrm{~d} \tau .
$$



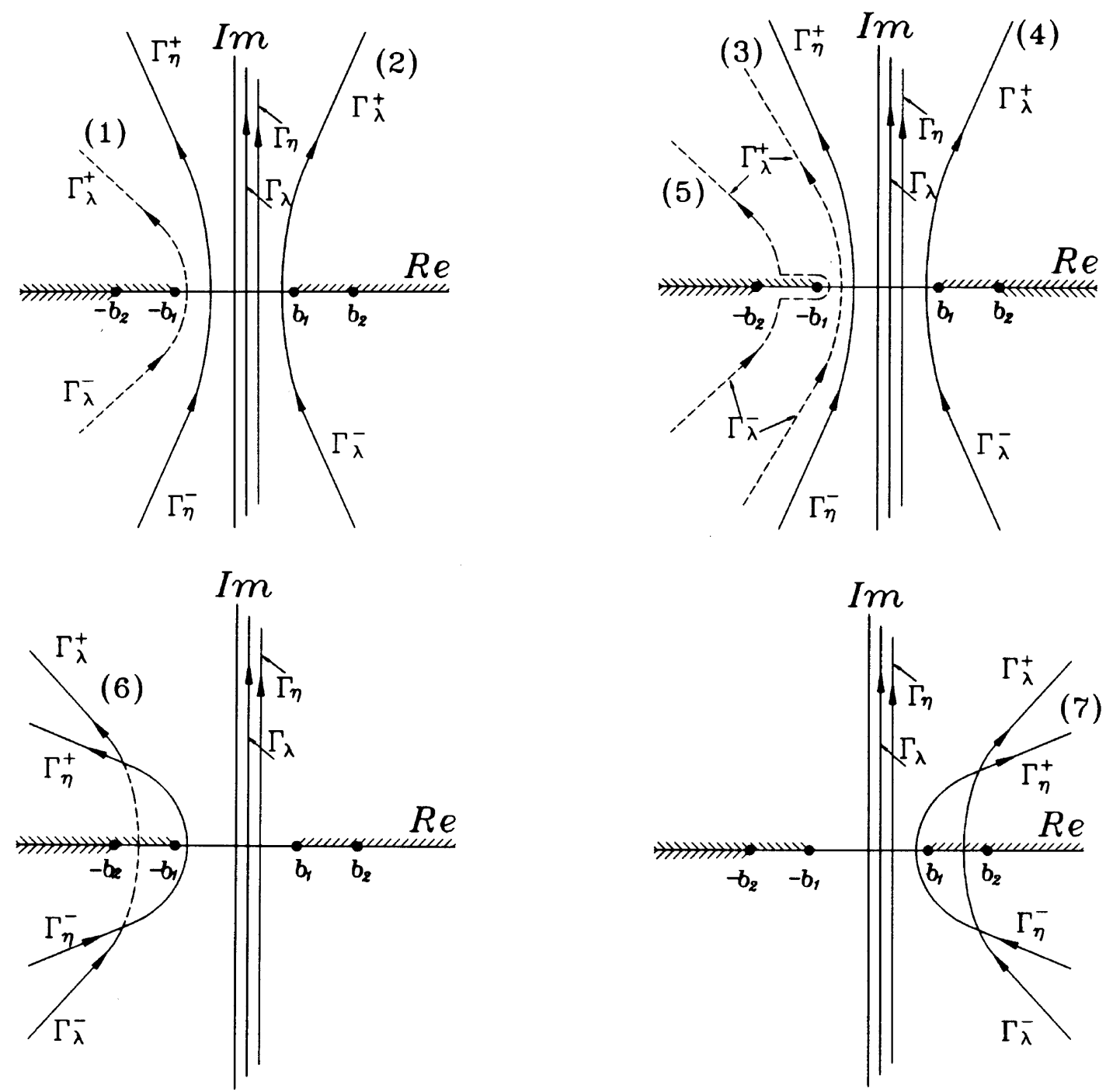

Fig. 3. (a) Deformed integral contour for conditions (1) and (2) of case 2. (b) Deformed integral contour for conditions (3), (4) and (5) of case 2. (c) Deformed integral contour for condition (6) of case 2. (d) Deformed integral contour for condition (7) of case 2 .

Case 2: $b_{1}<b_{2}$

$$
K_{\mathrm{III}}(t)=\sqrt{\frac{2}{\pi^{3}}} \int_{b_{1} R_{p}}^{t} \operatorname{Im}\left[\frac{f_{1 / 2}(\eta) S_{1}^{+}(\eta)}{\left(b_{1}+\eta\right)^{1 / 2}} \frac{\partial \eta}{\partial \tau}\right] \frac{1}{(t-\tau)^{1 / 2}} \mathrm{~d} \tau .
$$

For the special case of homogeneous medium, i.e. $b_{1}=b_{2}$ and $\mu_{1}=\mu_{2}$, the solution of stress intensity factor can be reduced to that obtained by Ma and Chen (1993) as follows 


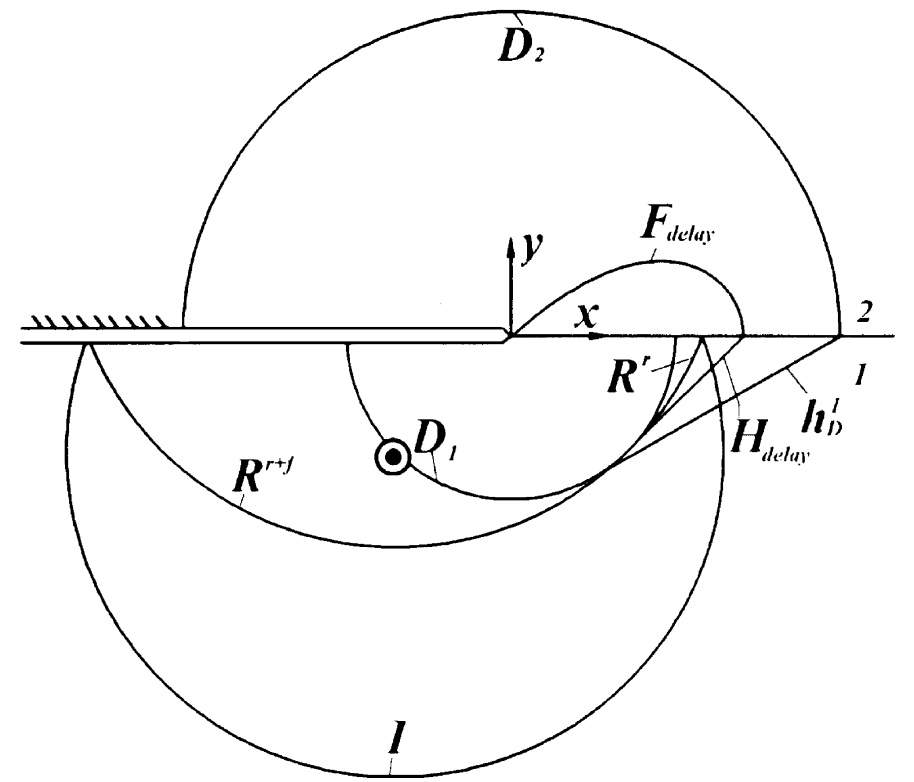

Fig. 4. Wave fronts of the incident, reflected, refracted and diffracted waves with loading applied at $\theta_{p}=45^{\circ}$ and $c_{2} / c_{1}=2$ for case 1 .

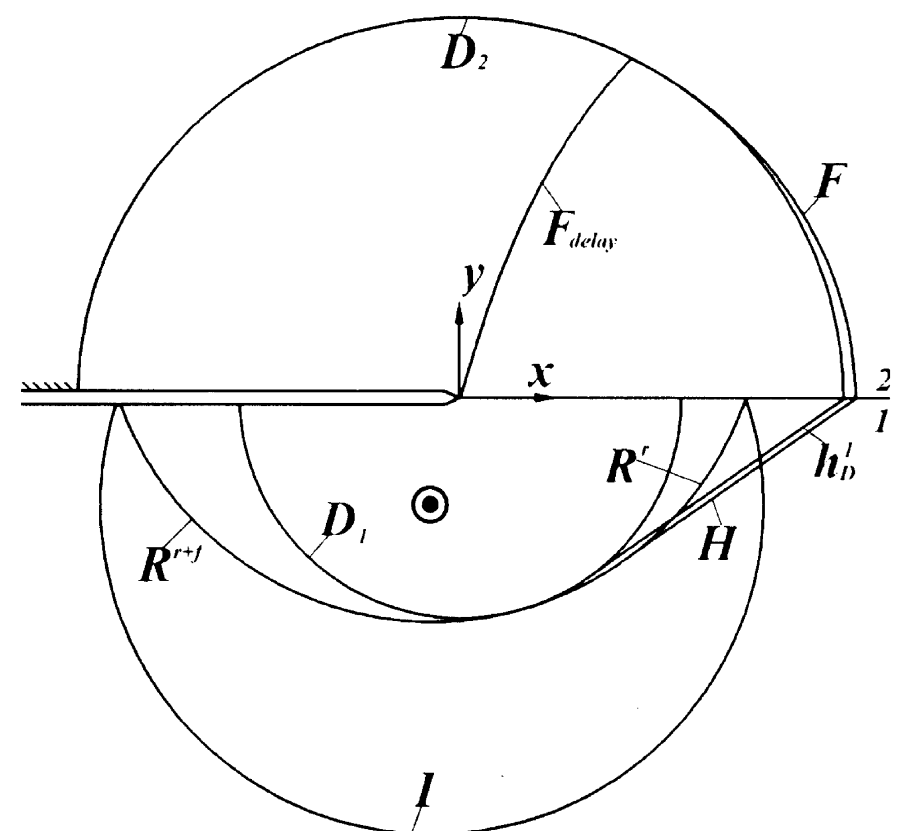

Fig. 5. Wave fronts of the incident, reflected, refracted and diffracted waves with loading applied at $\theta_{p}=75^{\circ}$ and $c_{2} / c_{1}=\sqrt{3}$ for case 1 . 


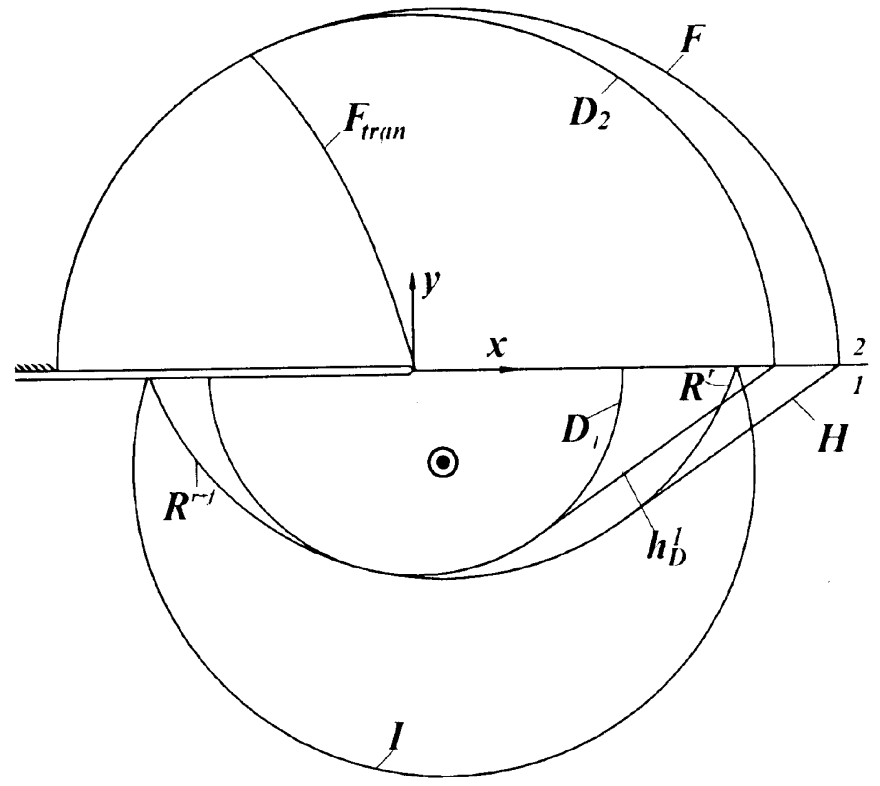

Fig. 6. Wave fronts of the incident, reflected, refracted and diffracted waves with loading applied at $\theta_{p}=105^{\circ}$ and $c_{2} / c_{1}=\sqrt{3}$ for case 1 .

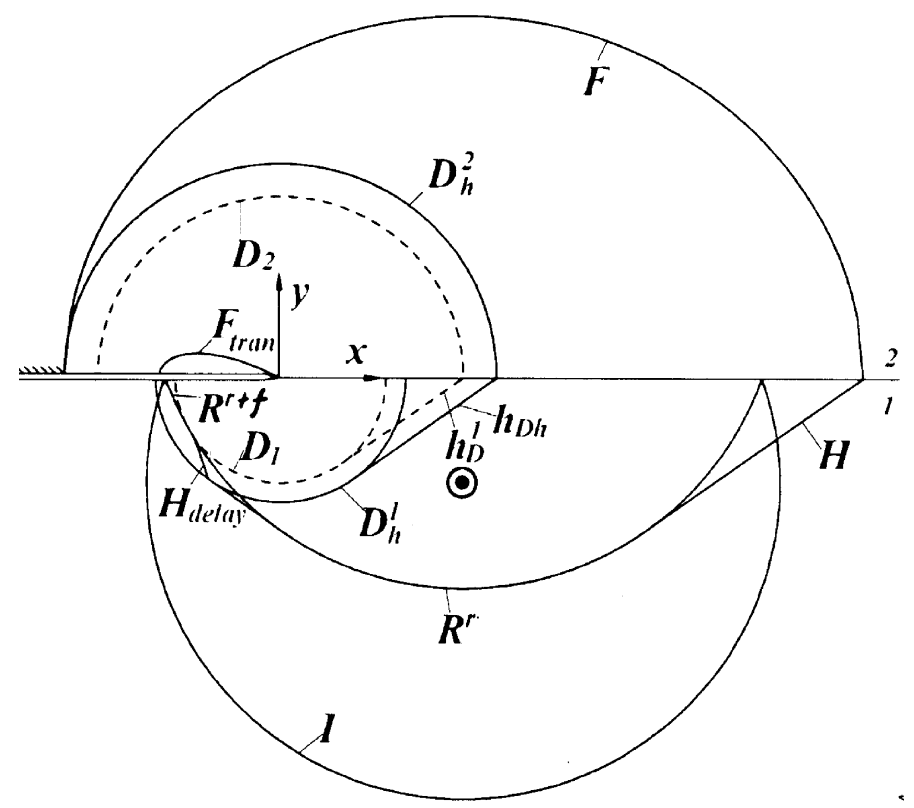

Fig. 7. Wave fronts of the incident, reflected, refracted and diffracted waves with loading applied at $\theta_{p}=150^{\circ}$ and $c_{2} / c_{1}=\sqrt{3}$ for case 1 . 


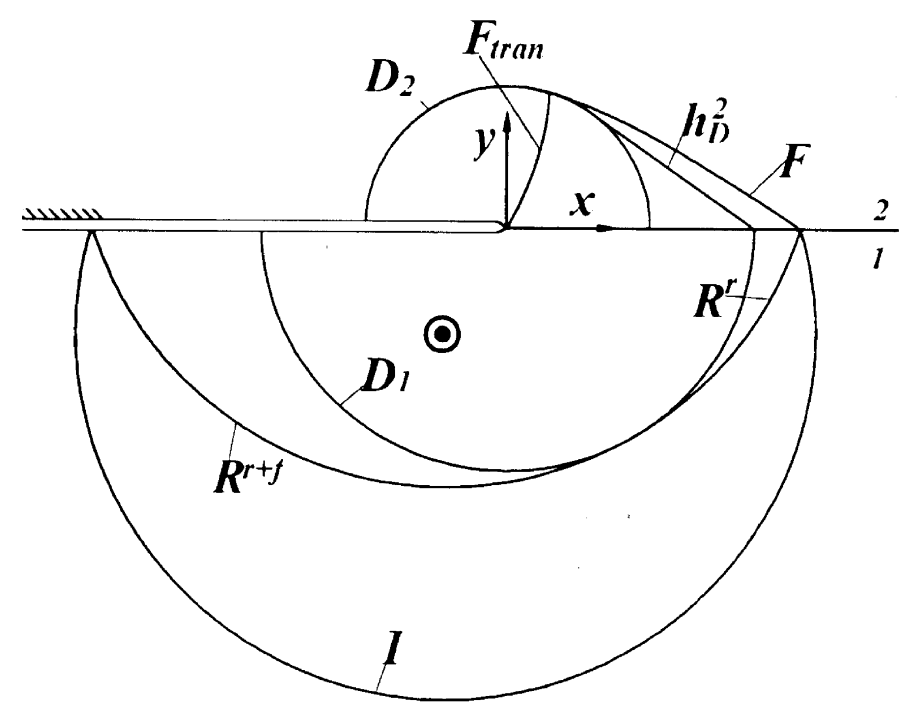

Fig. 8. Wave fronts of the incident, reflected, refracted and diffracted waves with loading applied at $\theta_{p}=60^{\circ}$ and $c_{2} / c_{1}=1 / \sqrt{3}$ for case 2 .

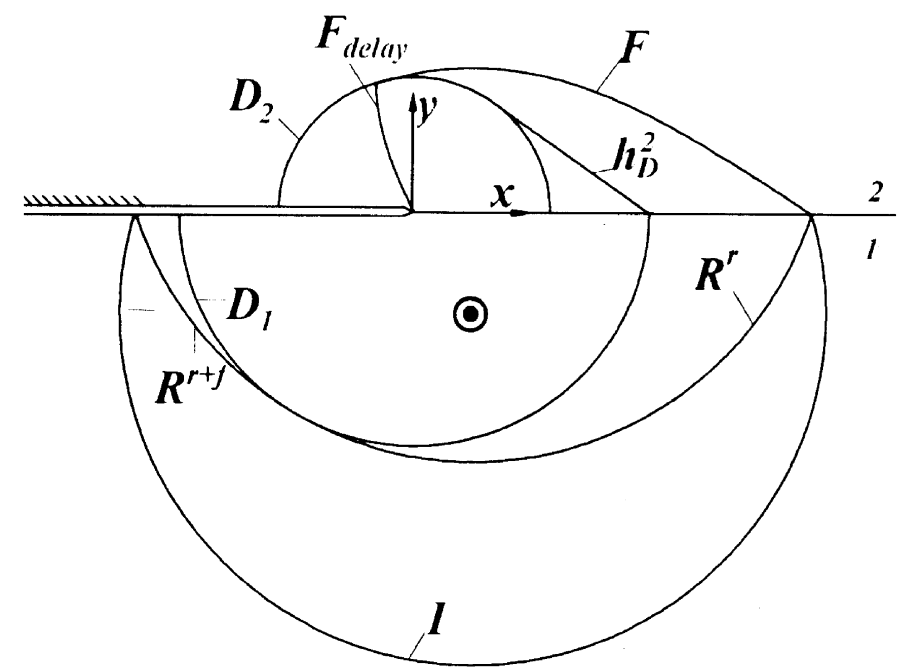

Fig. 9. Wave fronts of the incident, reflected, refracted and diffracted waves with loading applied at $\theta_{p}=120^{\circ}$ and $c_{2} / c_{1}=1 / \sqrt{3}$ for case 2 . 


$$
\begin{aligned}
K_{\mathrm{III}}^{0} & =\sqrt{\frac{2}{\pi^{3}}} \int_{b_{1} R_{p}}^{t} \operatorname{Im}\left[\frac{\partial \eta / \partial \tau}{\left(b_{2}+\eta\right)^{1 / 2}}\right] \frac{1}{(t-\tau)^{1 / 2}} \mathrm{~d} \tau \\
& =\sqrt{\frac{2}{\pi R_{p}}} \cos \left(\frac{\theta_{p}}{2}\right) H(t-b R) .
\end{aligned}
$$

The interesting result represented in (53) is that the dynamic stress intensity factor for a semiinfinite crack on a homogeneous medium jumps from zero to the static value after the incident shear wave generated from the loading point arrives at the crack tip. The corresponding static value for a semi-infinite interfacial crack subjected to a static body force is

$$
K_{\mathrm{III}}^{s}=\frac{2 \mu_{2}}{\mu_{1}+\mu_{2}} K_{\mathrm{III}}^{0}=\frac{2 \mu_{2}}{\mu_{1}+\mu_{2}} \sqrt{\frac{2}{\pi R_{p}}} \cos \left(\frac{\theta_{p}}{2}\right) .
$$

\section{Numerical results}

The explicit analytical transient results for the stress $\sigma_{y z}$ and the stress intensity factor have been given in the previous section. The transient response of stress for an applied concentrated force with Heaviside function dependence at the position $r=R_{p}$ and $\theta=\theta_{p}=45^{\circ}$ is investigated first. The transient stress is evaluated at two field points A and B as shown in Fig. 1, in which A ( $\left.R_{d_{1}}=0.4 h, \theta_{d_{1}}=20^{\circ}\right)$ is in material 1 and $\mathrm{B}\left(R_{d_{2}}=0.4 h, \theta_{d_{2}}=20^{\circ}\right)$ is in material 2. Figure 10

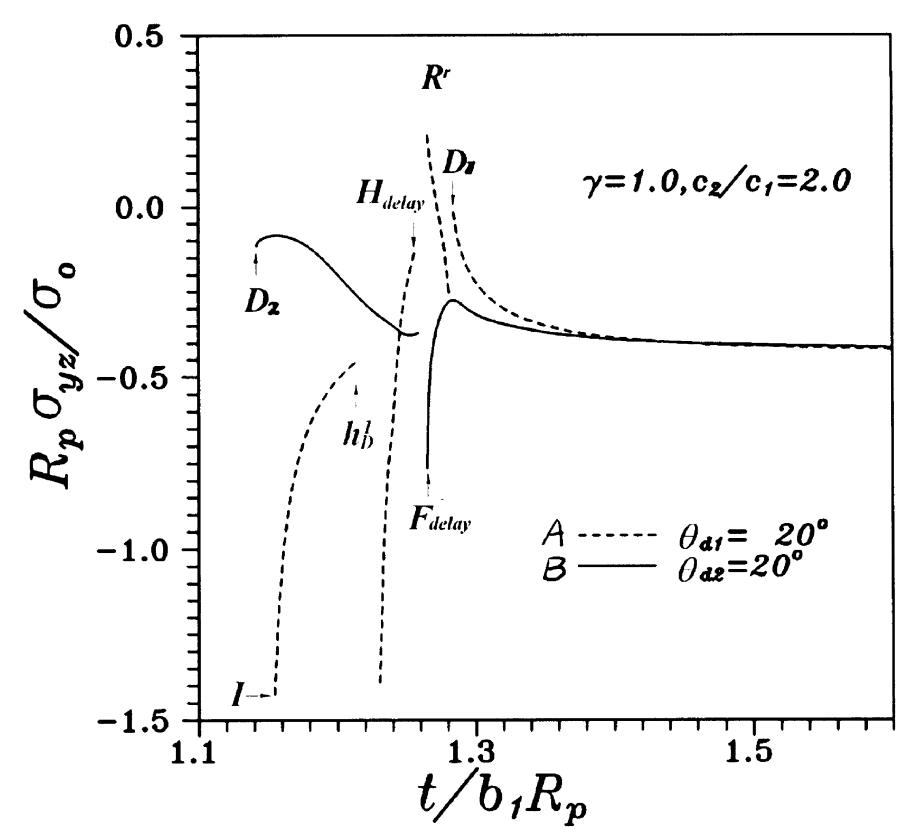

Fig. 10. Transient stress $\sigma_{y z}$ at field points A and B subjected to concentrated force applied at $\theta_{p}=45^{\circ}$ and $c_{2} / c_{1}=2$. 


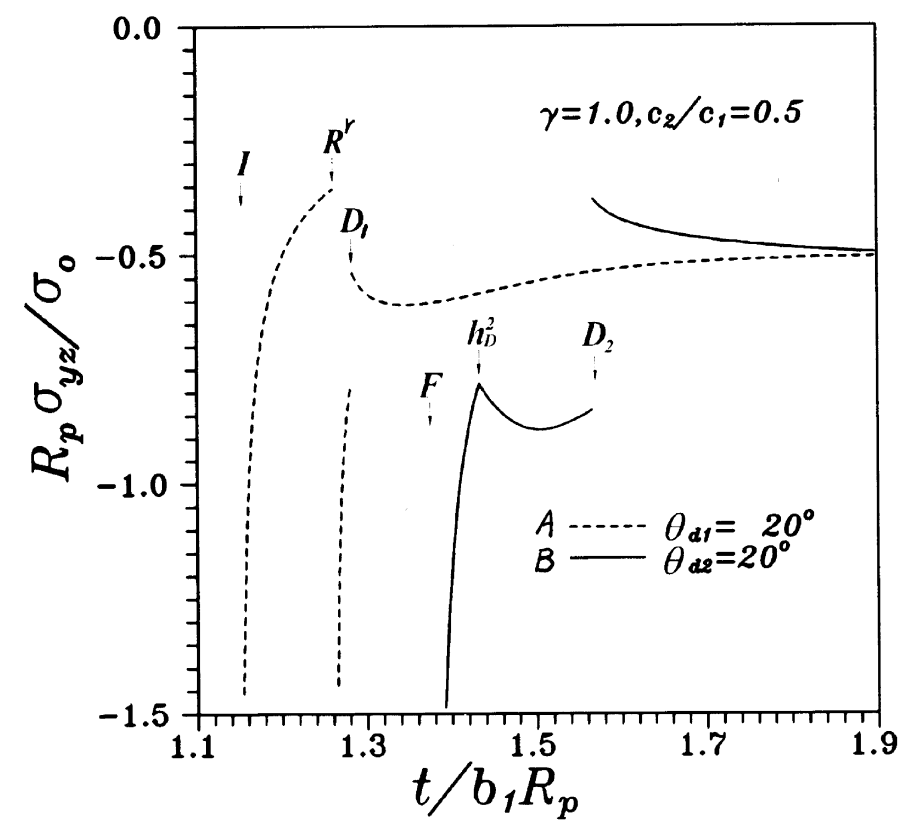

Fig. 11. Transient stress $\sigma_{y z}$ at field points A and B subjected to concentrated force applied at $\theta_{p}=45^{\circ}$ and $c_{2} / c_{1}=0.5$.

shows the transient response of stress for the case of $\gamma=\mu_{2} / \mu_{1}=1$ and $c_{2} / c_{1}=2$, the corresponding complete wave fronts are shown in Fig. 4 . The time has been normalized by dividing by $b_{1} R_{p}$ and the arrival time for each wave front is indicated in the figure. Figure 11 shows the transient response of stress for the case of $\gamma=1$ and $c_{2} / c_{1}=0.5$, the complete wave fronts are similar to Fig. 8. It is worthy to indicate that after the last wave has passed the field point, the value of transient stresses will tend toward the corresponding static result.

The dynamic stress intensity factors for different situations are shown in Figs 12-15. The dynamic stress intensity factor has been normalized by the corresponding result of the homogeneous case $K_{\mathrm{III}}^{0}$. Figure 12 shows an interesting result that the dynamic stress intensity factor will jump to the corresponding static value for the special case of $c_{1}=c_{2}$. For the case of $\mu_{1}=\mu_{2}$, the dynamic stress intensity factors for different ratio of $c_{2} / c_{1}$ are presented in Fig. 13. It shows in Fig. 13 that the dynamic stress intensity factor will decrease and approach to a constant value for $c_{1}>c_{2}$, but increase and approach to a constant value for $c_{1}<c_{2}$. The transient history of the dynamic stress intensity factor for applying a point loading at different location for $\mu_{2} / \mu_{1}=1.5$ and $c_{2} / c_{1}=1.2$ is shown in Fig. 14. Figure 15 shows the result for the case of $\mu_{2} / \mu_{1}=0.75$ and $c_{2} / c_{1}=0.9$. It is of interest to see that the dynamic stress intensity factor will be larger than the homogeneous case in the transient period for $\mu_{2}>\mu_{1}$ and $c_{2}>c_{1}$ (Fig. 14), and will be smaller than the homogeneous case for $\mu_{2}<\mu_{1}$ and $c_{2}<c_{1}$ (Fig. 15).

\section{Conclusions}

Most of the problems that have been studied in the development of fracture mechanics are quasi-static. Numerous problems have existed for which the assumption that the deformation is 


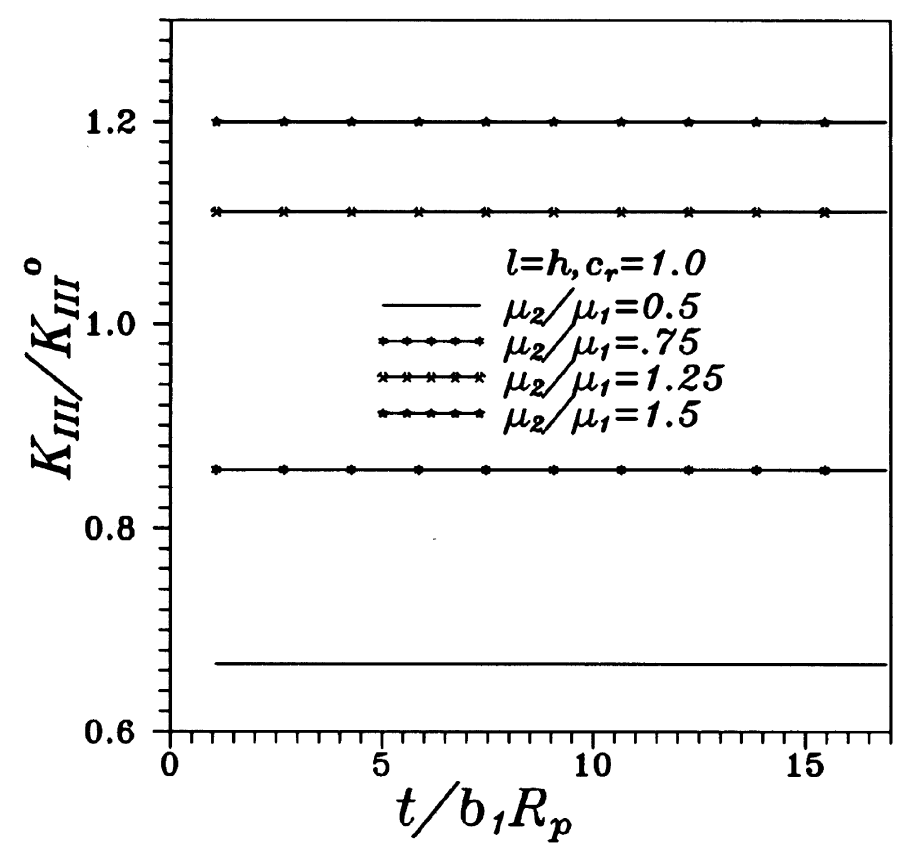

Fig. 12. Dynamic stress intensity factors for $c_{2} / c_{1}=1, \theta_{p}=45^{\circ}$ and different values of $\mu_{2} / \mu_{1}$.

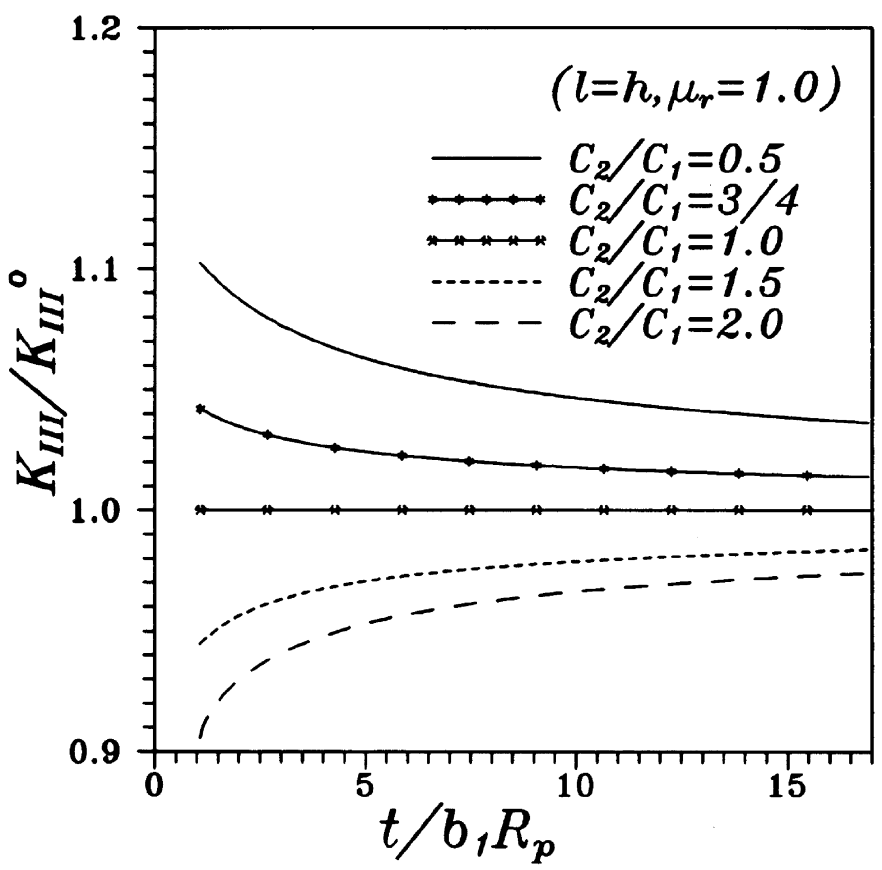

Fig. 13. Dynamic stress intensity factors for $\mu_{2} / \mu_{1}=1, \theta_{p}=45^{\circ}$ and different values of $c_{2} / c_{1}$. 


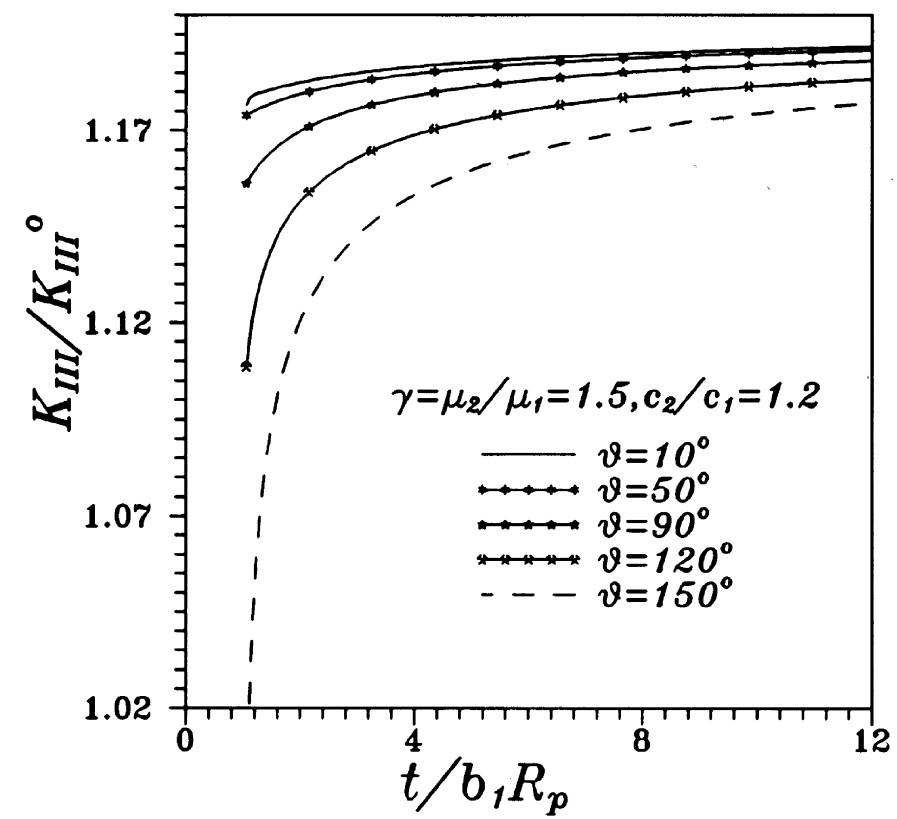

Fig. 14. Dynamic stress intensity factors for $\mu_{2} / \mu_{1}=1.5, c_{2} / c_{1}=1.2$ and different values of $\theta_{p}$.

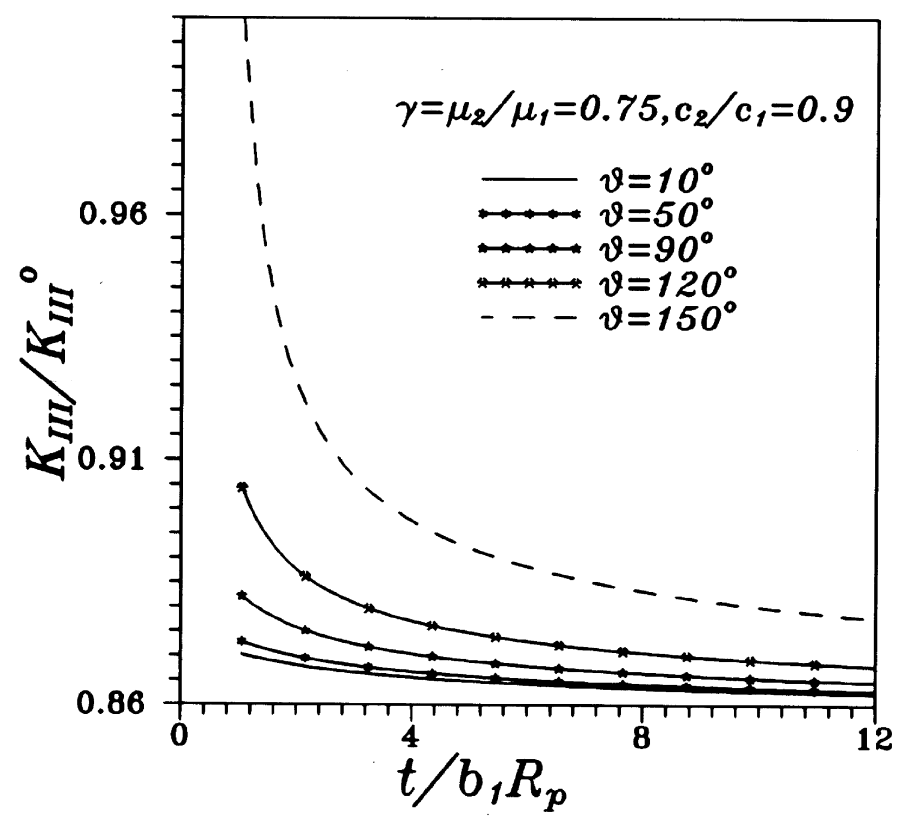

Fig. 15. Dynamic stress intensity factors for $\mu_{2} / \mu_{1}=0.75, c_{2} / c_{1}=0.9$ and different values of $\theta_{p}$. 
quasi-static is invalid and the inertia of the material must be taken into account. The mechanical behavior of many newly developed multiphase materials are mainly controlled by the response of the interface. Many researchers have devoted to investigating the field of dynamic debonding along a bimaterial interface. The transient problem of a semi-infinite interface crack in an infinite bimaterial is considered in this study. The equivalent static problem has been studied by many investigators in the past twenty years, but the transient solution is very few. In conventional studies of a semi-infinite crack in a homogeneous unbounded medium subjected to dynamic loading, the complete solution can be obtained by applying direct integral transform methods. If a dynamic cracked problem having a characteristic length or the loading condition is unsymmetric, then the same procedure cannot be applied directly. In this paper, the transient full field solutions and dynamic stress intensity factor of a semi-infinite interface crack in an infinite bimaterial subjected to a concentrated loading applied on the bimaterial medium is obtained. The present results have been justified by the corresponding static value and homogeneous case. These transient solutions are obtained by superposition of a proposed fundamental solution in the Laplace transform domain. The proposed fundamental solution is an exponentially distributed traction applied on the crack faces. This fundamental solution is successfully applied towards solving this transient problem and is demonstrated as an efficient methodology to solve other similar problems. The exact solution of this configuration can provide a valuable check for pure numerical methods such as finite element, finite difference or boundary element method in solving more complex geometries.

Some interesting and important results are obtained in this study. We found that the dynamic stress intensity factor will be larger than homogeneous case if the loading is applied at the softer material. However if the loading is applied at the harder material of the bimaterial medium, then the dynamic stress intensity factor in the whole transient period will be smaller than the corresponding homogeneous case. The results obtained in this investigation provide much information that is very important for the study on dynamic fracture. The powerful technique used in this paper can be provided for further investigation in more complicated dynamic fracture problems especially on the crack propagation event. Moreover, it is easy to extend the method proposed here to solve more difficult problems which involve interaction of a finite crack with boundaries. A further study related to dynamic fracture based on this method will be given in a follow-up report.

\section{Acknowledgements}

The authors gratefully acknowledge the financial support of this research by the National Science Council (Republic of China) under Grant NSC 84-2212-E001-062.

\section{Appendix}

The complete transient solutions for incident wave, reflected wave, refracted wave and diffracted wave expressed in time domain for shear stress $\sigma_{y z}$ are shown as follows 
Material 1:

\begin{tabular}{|c|c|}
\hline Wave & Transient solution \\
\hline$I$ & $\frac{-1}{\pi} \operatorname{Im}\left[\frac{\partial \eta^{+}}{\partial t_{i}}\right] H\left(t_{i}-b_{1} R_{i}\right)$ \\
\hline$R^{r}$ & $\frac{1}{\pi} \operatorname{Im}\left[r_{1 / 2}(\eta) \frac{\partial \eta^{+}}{\partial t_{r}}\right] H\left(t_{r}-b_{1} R_{r}\right)$ \\
\hline$R^{r+f}$ & $\frac{1}{\pi} \operatorname{Im}\left[\frac{\partial \eta^{+}}{\partial t_{r}}\right] H\left(t_{r}-b_{1} R_{r}\right)$ \\
\hline$H$ & $\frac{1}{\pi} \operatorname{Im}\left[r_{1 / 2}\left(\eta_{0}^{+}\right) \frac{\partial \eta_{0}^{+}}{\partial t_{r}}\right]\left[H\left(t_{r}-T_{h_{r}}\right)-H\left(t_{r}-b R_{r}\right)\right]$ \\
\hline$H_{\text {delay }}$ & $\frac{1}{\pi} \operatorname{Im}\left[r_{1 / 2}\left(\eta_{0}^{+}\right) \frac{\partial \eta_{0}^{+}}{\partial t_{r}}\right]\left[H\left(t_{r}-T_{h_{d}}\right)-H\left(t_{r}-b_{1} R_{r}\right)\right]$ \\
\hline$H_{\text {delay }}^{c}$ & $\frac{1}{\pi} \operatorname{Im}\left[r_{1 / 2}\left(\eta_{0}^{+}\right) \frac{\partial \eta_{0}^{+}}{\partial t_{r}}\right]\left[H\left(t_{r}-T_{h_{d}}^{\prime}\right)-H\left(t_{r}-b_{1} R_{r}\right)\right]$ \\
\hline$D_{1}$ & $-\frac{1}{2 \pi^{2}} \int_{b_{1} R_{p}}^{t-b_{1} R_{d_{1}}} \operatorname{Re}\left[H\left(\lambda^{+}, \eta^{+}\right) \frac{\partial \lambda^{+}}{\partial t_{d_{1}}} \frac{\partial \eta^{+}}{\partial t_{p}}-H\left(\lambda^{-}, \eta^{+}\right) \frac{\partial \lambda^{-}}{\partial t_{d_{1}}} \frac{\partial \eta^{+}}{\partial t_{p}}\right] \mathrm{d} t_{p}$ \\
\hline$h_{D}^{1}$ & $-\frac{1}{2 \pi^{2}} \int_{t-b_{1} R_{d_{1}}}^{t-T_{h_{d_{1}}}} \operatorname{Re}\left[H\left(\lambda_{h}^{+}, \eta^{+}\right) \frac{\partial \lambda_{h}^{+}}{\partial t_{d_{1}}} \frac{\partial \eta^{+}}{\partial t_{p}}-H\left(\lambda_{h}^{-}, \eta^{+}\right) \frac{\partial \lambda_{h}^{-}}{\partial t_{d_{1}}} \frac{\partial \eta^{+}}{\partial t_{p}}\right] H\left(t_{p}-b_{1} R_{p}\right) \mathrm{d} t_{p}$ \\
\hline$D_{h}^{1}$ & $-\frac{1}{2 \pi^{2}} \int_{T_{h_{p}}}^{b_{1} R_{p}} \operatorname{Re}\left[H\left(\lambda^{+}, \eta^{+}\right) \frac{\partial \lambda^{+}}{\partial t_{d_{1}}} \frac{\partial \eta_{h}^{+}}{\partial t_{p}}-H\left(\lambda^{-}, \eta_{h}^{+}\right) \frac{\partial \lambda^{-}}{\partial t_{d_{1}}} \frac{\partial \eta_{h}^{+}}{\partial t_{p}}\right] H\left(t-b_{1} R_{d_{1}}-t_{p}\right) \mathrm{d} t_{p}$ \\
\hline$h_{D_{h}}^{1}$ & $\begin{array}{l}-\frac{1}{2 \pi^{2}} \int_{T_{h_{p}}}^{b_{1} R_{p}} \operatorname{Re}\left[H\left(\lambda_{h}^{+}, \eta_{h}^{+}\right) \frac{\partial \lambda_{h}^{+}}{\partial t_{d_{1}}} \frac{\partial \eta_{h}^{+}}{\partial t_{p}}-H\left(\lambda_{h}^{-}, \eta_{h}^{+}\right) \frac{\partial \lambda_{h}^{-}}{\partial t_{d_{1}}} \frac{\partial \eta_{h}^{+}}{\partial t_{p}}\right] H\left(t-T_{h_{d_{1}}}-t_{p}\right) \mathrm{d} t_{p} \\
+\frac{1}{2 \pi^{2}} \int_{T_{h_{p}}}^{b_{1} R_{p}} \operatorname{Re}\left[H\left(\lambda_{h}^{+}, \eta_{h}^{+}\right) \frac{\partial \lambda_{h}^{+}}{\partial t_{d_{1}}} \frac{\partial \eta_{h}^{+}}{\partial t_{p}}-H\left(\lambda_{h}^{-}, \eta_{h}^{+}\right) \frac{\partial \lambda_{h}^{-}}{\partial t_{d_{1}}} \frac{\partial \eta_{h}^{+}}{\partial t_{p}}\right] H\left(t-b_{1} R_{d_{1}}-t_{p}\right) \mathrm{d} t_{p}\end{array}$ \\
\hline
\end{tabular}

Material 2

\begin{tabular}{ll}
\hline$F$ & $-\frac{1}{\pi} \operatorname{Im}\left[f_{1 / 2}(\eta) \frac{\partial \eta^{+}}{\partial t_{f}}\right] H\left(t_{f}-T_{f}\right)$ \\
$D_{2}$ & $-\frac{1}{2 \pi^{2}} \int_{b_{1} R_{p}}^{t-b R_{d_{2}}} \operatorname{Re}\left[H\left(\lambda^{+}, \eta^{+}\right) \frac{\partial \lambda^{+}}{\partial t_{d_{2}}} \frac{\partial \eta^{+}}{\partial t_{p}}-H\left(\lambda^{-}, \eta^{+}\right) \frac{\partial \lambda^{-}}{\partial t_{d_{2}}} \frac{\partial \eta^{+}}{\partial t_{p}}\right] \mathrm{d} t_{p}$ \\
$D_{h}^{2}$ & $-\frac{1}{2 \pi^{2}} \int_{T_{h_{p}}}^{b_{1} R_{p}} \operatorname{Re}\left[H\left(\lambda^{+}, \eta_{h}^{+}\right) \frac{\partial \lambda^{+}}{\partial t_{d_{2}}} \frac{\partial \eta_{h}^{+}}{\partial t_{p}}-H\left(\lambda^{-}, \eta_{h}^{+}\right) \frac{\partial \lambda^{-}}{\partial t_{d_{2}}} \frac{\partial \eta_{h}^{+}}{\partial t_{p}}\right] H\left(t-b_{2} R_{d_{2}}-t_{p}\right) \mathrm{d} t_{p}$ \\
$F_{\text {delay }}$ & $-\frac{1}{\pi} \operatorname{Im}\left[f_{1 / 2}(\eta) \frac{\partial \eta^{+}}{\partial t_{f}}\right] H\left(t_{f}-T_{f^{\prime}}\right)$ \\
$F_{\text {tran }}$ & $-\frac{1}{\pi} \operatorname{Im}\left[f_{1 / 2}(\eta) \frac{\partial \eta^{+}}{\partial t_{f}}\right]\left[H\left(t_{f}-T_{f}\right)-H\left(t_{f}-T_{f^{\prime}}\right)\right]$ \\
$h_{D}^{2}$ & $-\frac{1}{2 \pi^{2}} \int_{t-b_{2} R_{d_{2}}}^{t-T_{h_{d_{2}}}} \operatorname{Re}\left[H\left(\lambda^{+}, \eta_{h_{2}}^{+}\right) \frac{\partial \lambda^{+}}{\partial t_{d_{2}}} \frac{\partial \eta_{h_{2}}^{+}}{\partial t_{p}}-H\left(\lambda^{-}, \eta_{h_{2}}^{+}\right) \frac{\partial \lambda^{-}}{\partial t_{d_{2}}} \frac{\partial \eta_{h_{2}}^{+}}{\partial t_{p}}\right] H\left(t_{p}-b_{1} R_{p}\right) \mathrm{d} t_{p}$ \\
\hline
\end{tabular}


where

$$
\begin{aligned}
& \lambda_{0}^{ \pm}=\frac{-t_{r} \cos \theta_{r}}{R_{r}}+\frac{\sin \theta_{r}}{R_{r}}\left(b_{1}^{2} R_{r}^{2}-t_{r}^{2}\right)^{1 / 2} \pm i \varepsilon \\
& \eta_{h}^{ \pm}=\frac{-t_{p} \cos \theta_{p}}{R_{p}}+\frac{\sin \theta_{p}}{R_{p}}\left(b_{1}^{2} R_{p}^{2}-t_{p}^{2}\right)^{1 / 2} \pm i \varepsilon \\
& \lambda_{h}^{ \pm}=\frac{-t_{d_{1}} \cos \theta_{d_{1}}}{R_{d_{1}}}+\frac{\sin \theta_{d_{1}}}{R_{d_{1}}}\left(b_{1}^{2} R_{d_{1}}^{2}-t_{d_{1}}^{2}\right)^{1 / 2} \pm i \varepsilon \\
& \lambda_{h_{2}}^{ \pm}=\frac{-t_{d_{2}} \cos \theta_{d_{2}}}{R_{d_{2}}}+\frac{\sin \theta_{d_{2}}}{R_{d_{2}}}\left(t_{d_{2}}^{2}-b_{2}^{2} R_{d_{2}}^{2}\right)^{1 / 2} \pm i \varepsilon \\
& T_{h_{r}}=b_{2}(x+l)-\left(b_{1}^{2}-b_{2}^{2}\right)^{1 / 2}(y-h) \\
& T_{h_{d_{1}}}=b_{2} x-\left(b_{1}^{2}-b_{2}^{2}\right)^{1 / 2} y \\
& T_{h_{p}}=b_{2} l-\left(b_{1}^{2}-b_{2}^{2}\right)^{1 / 2} h \\
& T_{h_{d_{2}}}=b_{2} x+\left(b_{2}^{2}-b_{1}^{2}\right)^{1 / 2} y \\
& T_{h_{d}}=b_{1} R_{p}+b_{1} x \cos \theta_{p}-b_{1} y \sin \theta_{p} \\
& T_{f^{\prime}}=\left[R_{p}\left|\cos \theta_{d_{2}}\right|+R_{d_{2}}\left|\cos \theta_{p}\right|\right] \sqrt{\frac{b_{1}^{2} \sin ^{2} \theta_{p}-b_{2}^{2} \sin ^{2} \theta_{d_{2}}}{\sin ^{2} \theta_{p}-\sin ^{2} \theta_{d_{2}}}} \\
& T_{h_{d}}^{\prime}=b_{1} R_{d_{1}}+b_{1} l \cos \theta_{d_{1}}+b_{1} h \sin \theta_{d_{1}} .
\end{aligned}
$$

\section{References}

Bogy, D. B. (1972) The plane solution for anisotropic elastic wedges under normal and shear loading. Journal of Applied Mechanics 39, 1103-1109.

Brock, L. M. and Achenbach, J. D. (1973) Extension of an interface flaw under the influence of transient waves. International Journal of Solids and Structures 9, 53-68.

Brock, L. M. (1982) Shear and normal impact loading on one face of a narrow slip. International Journal of Solids and Structures 18, 467-477.

Brock, L. M. (1984) Stresses in a surface obstacle undercut due to rapid indentation. Journal of Elasticity 14, 415-424.

Brock, L. M., Jolles, M. and Schroedl, M. (1985) Dynamic impact over a subsurface crack: applications to the dynamic tear test. Journal of Applied Mechanics 52, 287-290.

de Hoop, A. T. (1958) Representation theorems for the displacement in an elastic solid and their application to elastodynamic diffraction theory. Doctoral dissertation, Technische hoegschool, Delft.

England, A. H. (1965) A crack between dissimilar media. Journal of Applied Mechanics 32, 400-402.

Erdogan, F. (1965) Stress distribution in bonded dissimilar material with cracks. Journal of Applied Mechanics 32, 403410.

Freund, L. B. (1972a) Crack propagation in an elastic solid subjected to general loading-I. Constant rate of extension. Journal of Mechanics and Physics of Solids 20, 129-140.

Freund, L. B. (1972b) Crack propagation in an elastic solid subjected to general loading-II. Non-uniform rate of extension. Journal of Mechanics and Physics of Solids 20, 141-152.

Freund, L. B. (1973) Crack propagation in an elastic solid subjected to general loading-III. Stress wave loading. Journal of Mechanics and Physics of Solids 21, 47-61. 
Freund, L. B. (1974a) Crack propagation in an elastic solid subjected to general loading-IV. Obliquely incident stress pulse. Journal of Mechanics and Physics of Solids 22, 137-146.

Freund, L. B. (1974b) The stress intensity factor due to normal impact loading of the faces of a crack. International Journal of Engineering Science 12, 179-189.

Gotoh, H. (1967) Some problems of bonded anisotropic plates with cracks along the bond. International Journal of Fracture 3, 253-265.

Harris, J. G. (1980) Diffraction by a crack of a cylindrical longitudinal pulse. Journal of Applied Mathematics and Physics 31, 367-383.

Kuo, M. C. and Bogy, D. B. (1974) Plane solutions for the displacement and traction-displacement problems for anisotropic elastic wedges. Journal of Applied Mechanics 41, 197-202.

Ma, C. C. (1988) Initiation, propagation, and kinking of an in-plane crack. Journal of Applied Mechanics 55, 587-595.

Ma, C. C. (1990) Dynamic mixed mode I-II crack kinking under oblique stress wave loading in brittle solids. Journal of Applied Mechanics 57, 117-127.

Ma, C. C. and Burgers, P. (1986) Mode III kinking with delay time: an analytical approximation. International Journal of Solids and Structures 22, 883-899.

Ma, C. C. and Burgers, P. (1987) Dynamic mode I and mode II crack kinking including delay time effects. International Journal of Solids and Structures 23, 897-918.

Ma, C. C. and Burgers, P. (1988) Initiation, propagation, and kinking of an antiplane crack. Journal of Applied Mechanics 55, 111-119.

Ma, C. C. and Chen, S. K. (1993) Exact transient analysis of an anti-plane semi-infinite crack subjected to dynamic body forces. Wave Motion 17, 161-171.

Ma, C. C. and Hou, Y. C. (1990) Theoretical analysis of the transient response for a stationary inplane crack subjected to dynamic impact loading. International Journal of Engineering Science 28, 1321-1329.

Ma, C. C. and Hou, Y. C. (1991) Transient analysis for antiplane crack subjected to dynamic loadings. Journal of Applied Mechanics 58, 703-709.

Ma, C. C. and Ing, Y. S. (1995) Transient analysis of dynamic crack propagation with boundary effect. Journal of Applied Mechanics 62, 1029-1038.

Ma, C. C. and Luo, J. J. (1996) Plane solutions of interface cracks in anisotropic dissimilar media. Journal of Engineering Mechanics, ASCE 122, 30-38.

Noble, B. (1958) The Wiener-Hopf Technique. Pergamon Press.

Qu, J. and Bassani, J. L. (1989) Cracks on bimaterial and bicrystal interfaces. Journal of Mechanics and Physics of Solids 37, 417-433.

Rice, J. R. and Sih, G. C. (1965) Plane problems of cracks in dissimilar media. Journal of Applied Mechanics 32, 418423.

Ting, T. C. T. (1986) Explicit solution and invariance of the singularities at an interface crack in anisotropic composites. International Journal of Solids and Structures 22, 965-983.

Ting, T. C. T. (1990) Interface cracks in anisotropic bimaterials. Journal of Mechanics and Physics of Solids 38, 505513.

Tsai, C. H. and Ma, C. C. (1992) Transient analysis of a semi-infinite crack subjected to a dynamic concentrated force. Journal of Applied Mechanics 59, 804-811.

Williams, M. L. (1959) The stress around a fault or crack in dissimilar media. Bull. Seism. Soc. America 49, $199-204$. 